\title{
A Unified Design Framework for Single-Channel Dispersion-Managed Terrestrial Systems
}

\author{
Alberto Bononi, Paolo Serena, and Alessandra Orlandini
}

\begin{abstract}
This paper provides a unified framework to the design, performance optimization, and accurate numerical simulation of periodic, dispersion-managed (DM) single-channel long-haul optical transmission systems for nonsoliton on-off keying (OOK) modulation. The focus is on DM terrestrial systems, with identical spans composed of a long transmission fiber compensated at the span end by a linear dispersion compensating module, with pre- and postcompensation fibers at the beginning and end of the link. The framework is based on the dispersion-managed nonlinear Schrödinger equation (DM-NLSE). First, expressions of the DM-NLSE kernel are provided both in the frequency and the time domain, and a novel map strength parameter, appropriate for terrestrial systems, is introduced. It is then shown that the DM-NLSE contains all the basic information needed for system design, as summarized by three parameters: i) nonlinear phase, ii) in-line dispersion, and iii) map strength. Through a large-signal perturbative analysis of the DM-NLSE, the well-known linear relationship between the in-line dispersion and the optimal precompensation is derived, along with the large-signal step response of the DM link, from which the ghost pulses energy growth and a first estimation of the link memory are derived. The DM-NLSE is then linearized around the average signal field to get the amplitude/phase small-signal system matrix of the overall DM link, including pre- and postcompensation. By a singular-value decomposition of the small-signal DM link matrix, a novel expression of the memory of the optimized DM link is finally provided. Knowledge of such a memory is mandatory to run accurate numerical simulations and laboratory measurements with a sufficiently long pseudorandom bit sequence to avoid patterning effects.
\end{abstract}

Index Terms-Dispersion-managed nonlinear Schrödinger equation, optical fiber memory, perturbation methods, pseudorandom binary sequence (PRBS) length, small-signal analysis.

\section{INTRODUCTION}

$\mathbf{T}$ HE dispersion-managed (DM) nonlinear Schrödinger equation (NLSE) is a constant-coefficient partial differential equation describing the propagation of a polarized electric field along an ultralong DM periodic optical link [1]-[3]. It is derived from the standard NLSE using the method of multiple scales and represents an alternative mathematical derivation of the propagation equation previously obtained by the method of averaging for long-haul systems with periodic amplification

Manuscript received November 9, 2007; revised March 27, 2008. Current version published January 28, 2009. This work was supported by Alcatel-Lucent R\&I, France.

The authors are with the Dipartimento di Ingegneria dell'Informazione, Università degli Studi di Parma, 43100 Parma, Italy (e-mail: alberto.bononi@ unipr. it).

Color versions of one or more of the figures in this paper are available online at http://ieeexplore.ieee.org.

Digital Object Identifier 10.1109/JLT.2008.925030
[4], [5] or periodic amplification and dispersion [6]. A fundamental role in the DM-NLSE is played by the kernel, whose properties substantially determine the distortions induced on the propagating signals [2]. Since in the DM-NLSE each span represents a differential $d z$ [4], its applicability is in principle restricted to links with a very large number of spans, such as the ultralong submarine links with DM solitons for which it was initially developed.

In this paper, we are concerned instead with nonsoliton, long-and ultra-long-haul terrestrial systems with on-off keying (OOK) modulation, in which the number of spans need not be too large, typically ranging from 3 to 30 , and the period of the map equals the amplification period, with a dispersion-compensating fiber inserted inside the amplifier at the end of each span. Pre- and postcompensating fibers may also be present at the beginning and at the end of the link, respectively. The main purpose of this paper is to provide a unified framework to explain many valuable, empirically derived design rules for such systems and to derive new rules. Specifically, we will provide theoretical explanations of the following results.

i) The nonlinear cumulated phase plays a key role in setting performance of DM terrestrial systems [7].

ii) For a given nonlinear cumulated phase, the single parameter $-\beta_{2} R^{2} L_{\mathrm{eff}}$ is sufficient to determine system performance of optimized DM links, where $\beta_{2}$ is the group-velocity dispersion (GVD) parameter of the transmission fiber, $L_{\text {eff }}$ its effective length, and $R$ the bit-rate [8]-[12].

iii) A linear relationship between the optimal precompensation and the in-line dispersion, which we call the straight-line rule (SLR), exists for return-to-zero (RZ) OOK format [13], [14] and holds for non-RZ (NRZ) format as well [15], [16].

iv) For $40 \mathrm{~Gb} / \mathrm{s}$ DM systems with SLR-optimized precompensation, the optimal postcompensation scales linearly with nonlinear phase [17]. Moreover, we will prove the following novel result.

v) The minimum pseudorandom binary sequence (PRBS) length needed to run simulations that correctly account for intersymbol interference scales linearly with bit-rate in optimized periodic DM systems [18], unlike the quadratic growth found in the pseudolinear case dominated by GVD [19].

In this paper, it is shown that the DM-NLSE kernel contains most of the needed information for system optimization. For a DM terrestrial link, kernel expressions are provided both in the frequency and the time domains, and it is shown that they solely depend on the cumulated nonlinear phase mentioned at point i) and on (a slight variation of) the key parameter mentioned at point ii), which we call the map strength of the terrestrial link, 
whose connection with a similar definition for soliton systems is discussed in [20].

Both a method for computing the DM-NLSE and the approximation error of the DM-NLSE with respect to the NLSE was given in [21], where numerical results were provided for DM-soliton systems only. However, little insight can be obtained from [21] for nonsoliton limited-span DM systems. Hence, to explore the range of DM system parameters for which the DM-NLSE is applicable to our limited-span terrestrial systems, we provide extensive simulations of the NLSE based on the split-step Fourier method (SSFM) [22] and quantify the "distance" between the NLSE solution and the DM-NLSE solution. Since the optical signal-to-noise ratio (OSNR) penalty at a typical reference bit error rate (BER) (e.g., $10^{-5}$ in our case) is usually the performance parameter of interest to the design engineer, we provide OSNR penalty curves for a given set of DM parameters, computed for both the NLSE for a fixed number of spans and for the DM-NLSE, versus the nonlinear phase. From such curves at a selected strength, one finds that the $3 \mathrm{~dB}$ OSNR penalty is reached in the NLSE solution at a threshold phase, called the nonlinear threshold (NLT), which increases with the number of spans in the link, up to convergence to that of the DM-NLSE in the limit of an infinite-span system. This means that DM systems in which the same overall nonlinear phase is more evenly distributed along the link are more tolerant to nonlinear effects. Next, the accuracy of the DM-NLSE is shown to increase with map strength. For instance, in a fully compensated system with more than three spans, the DM-NLSE and NLSE estimated NLTs almost coincide for strengths larger than 0.08 - that is, for example, for $R>20 \mathrm{~Gb} / \mathrm{s}$ in links with transmission fiber dispersion of $8 \mathrm{ps} / \mathrm{nm} / \mathrm{km}$.

Having established the applicability range of the DM-NLSE to terrestrial systems, we next move to its analysis. Through a large-signal perturbative analysis, we first show analytically the origin of the well-known SLR mentioned at the above point iii) and explain the origin of the linear growth of the optimal postcompensation in SLR-optimized links mentioned at point iv).

We then derive analytical results on the large-signal step response of the DM link, from which a first appreciation of the link memory time and thus the intersymbol depth in OOK communications can be obtained. For OOK signals, we next linearize the DM-NLSE around the average signal field and obtain the small-signal power/phase system matrix of the overall DM link including pre- and postcompensation. Such a linearized system is indeed a generalization of the small-signal model developed in [16] for establishing the SLR and bears a certain resemblance with models already obtained in the single-span case [23], [24]. We show that the linearized model predictions are in good qualitative agreement with SSFM simulations and show over which parameters range the predictions are in a reasonable quantitative agreement. The linearized system can be used as a fast design tool to quickly perform comparisons among DM designs and obtain a rough estimate of system performance, similarly to the method used in [25], which is instead based on pulse broadening. By a singular value decomposition of the overall DM link matrix, including pre- and postcompensation fibers, we finally derive the novel expression of the memory of the DM link and thus the intersymbol depth mentioned at point v). Knowledge of such a memory is mandatory to run accurate numerical simulations and laboratory measurements with a sufficiently long pseudorandom bit sequence to avoid patterning effects. We discuss the dependence of memory on DM parameters and show that, in DM-optimized systems, the memory scales linearly with bit-rate instead of the quadratic growth found in GVD-dominated systems [19]. We finally discuss the impact of worst case bit-patterns on system performance and clarify that the well-known resonance of eye closure penalty versus map strength found through simulation [9]-[11] is indeed an artifact due to insufficient length of the employed PRBS. Please note that the single-channel optimal design rules presented in this paper may change substantially in wavelength-division multiplexing (WDM) transmissions at small map strength, where cross-channel effects largely dominate. The DM-NLSE approach can indeed be extended to WDM transmissions, and some results have already appeared in [26].

This paper is organized as follows. In Section II the DM-NLSE and the main aspects of the fiber kernel in terrestrial systems are given. In Section III, the numerical accuracy of the DM-NLSE is tested against systems with a finite number of spans. In Section IV, a first-order perturbative solution of the DM-NLSE and its use to derive the SLR are described. In Section V, a small-signal analysis of the DM-NLSE is provided. In Section VI, some interesting implications of the small-signal model like the memory of a DM optimized link are given. Section VII summarizes the main conclusions.

\section{THE DM-NLSE FOR TERRESTRIAL SYSTEMS}

A typical dispersion-managed terrestrial optical link is sketched in Fig. 1. Pre- and postcompensating fibers (pre- and postfiber, in brief) are placed before and after transmission, while the GVD of the transmission fiber is compensated by an in-line compensating fiber at each span end. The graph of the cumulated dispersion along the link is reported in the inset of Fig. 1. At the receiver, an optical bandpass filter (OBPF) selects the channel and after a quadratic detection a lowpass filter (LPF) brings the signal to the sampler.

The propagation of an electric field $A(z, t)$, with $z$ being the distance and $t$ the retarded time, is described by the NLSE (in engineering notation) as [22]

$$
\frac{\partial A}{\partial z}=j \frac{\beta_{2}(z)}{2} \frac{\partial^{2} A}{\partial t^{2}}-j \gamma(z)|A|^{2} A+\frac{g(z)}{2} A
$$

where $\beta_{2}(z)$ is the local group velocity dispersion parameter; $\gamma(z)$ is the local nonlinear coefficient; and $g(z)=-\alpha(z)+$ $\Sigma_{k} G_{k} \delta\left(z-z_{k}\right)$ is the power gain/attenuation coefficient, with $\alpha(z)$ being the local fiber attenuation, $\delta($.$) Dirac's delta func-$ tion, and $e^{G_{k}}$ the power gain of the $k$ th lumped amplifier of the link placed at $z_{k}=k L$, with $L$ the span length. In (1), we neglected the amplified spontaneous noise (ASE), whose impact was studied in [20] using the same analytical framework of this paper, and we assumed no polarization distortions. Dispersion 


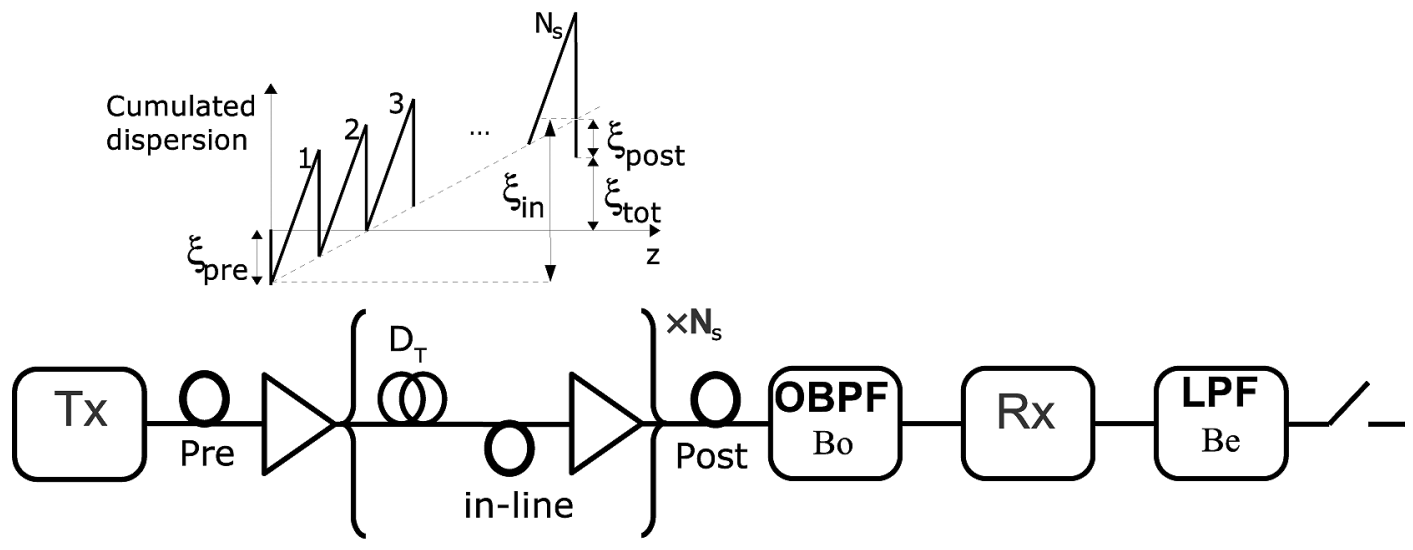

Fig. 1. System setup of the optical link. Inset: cumulative dispersion versus propagation distance $z$.

slope is also neglected. The reason is that, if the average dispersion slope is compensated at the end of each span, then distortions due to local dispersion slope can safely be neglected [11], [20]. From now on, we will focus on a single-channel transmission.

We now normalize the time to the supporting pulse duration $T$ of the transmitted signal by letting $\tau=t / T$. We also normalize the signal $A(z, t)$ to the power at coordinate $z$, i.e., $A(z, t)=$ $\sqrt{P} U(z, t) \exp \left((1 / 2) \int_{0}^{z} g(x) \mathrm{d} x\right)=\sqrt{P f(z)} U(z, t)$, being $f(z) \triangleq \exp \left(\int_{0}^{z} g(x) \mathrm{d} x\right)$ the net power gain from input to coordinate $z$, and $P$ a reference power. With these substitutions, (1) in the frequency domain becomes ${ }^{1}$

$$
\begin{aligned}
\frac{\partial \widetilde{U}(z, \omega)}{\partial z}= & -\frac{j \omega^{2} \widetilde{U}(z, \omega)}{2 L_{d}(z)}-\frac{j f(z)}{L_{\mathrm{n}}(z)} \iint \widetilde{U}\left(z, \omega+\omega_{1}\right) \\
& \times \widetilde{U}\left(z, \omega+\omega_{2}\right) \widetilde{U}^{*}\left(z, \omega+\omega_{1}+\omega_{2}\right) \frac{d \omega_{1}}{2 \pi} \frac{d \omega_{2}}{2 \pi}
\end{aligned}
$$

with the double integral extended to $\mathbb{R}^{2}$. In (2), we introduced the dispersion length $L_{d}(z) \triangleq T^{2} / \beta_{2}$ and the nonlinear length $L_{\mathrm{n}}(z) \triangleq 1 /(\gamma(z) P)$ [22]. Note that $L_{d}$ has the sign of $\beta_{2}$ and that $\omega /(2 \pi)$ is the frequency normalized to $1 / T$.

In [2] and [6], it was noted that in strongly dispersion-managed system, in which $\beta_{2}(z), \gamma(z)$, and $f(z)$ are all periodic in $z$ with period $L$, the GVD experiences large variations from its span-averaged value, a feature that suggests to write $\beta_{2}(z)=$ $\left\langle\beta_{2}\right\rangle+\Delta \beta_{2}(z)$, where, here and in the following, we define for any arbitrary function $g(z)$ its span-averaged value as $\langle g\rangle=$ $(1 / L) \int_{0}^{L} g(x) d x$. If also the nonlinearity per span is small, the periodic behavior of nonlinearity and GVD implies the existence of two different length scales in (1): a fast scale, i.e., for distances shorter than $L$, along which only the rapidly varying differential dispersion $\Delta \beta_{2}(z)$ significantly affects the electric field, and a slow scale over which the nonlinear effect and the average dispersion $\left\langle\beta_{2}\right\rangle$ mainly affect the field. Based on this observation, the authors in [2] suggested the use of a multiple-scale approach to approximate the solution of (2) at each span end

\footnotetext{
${ }^{1}$ The Fourier transform of a generic electric field $X(z, \tau)$ is defined as $\tilde{X}(z, \omega) \triangleq \int_{-\infty}^{\infty} X(z, \tau) e^{-j \omega \tau} d \tau$.
}

$z=n L, n \in \mathbb{N}$, with the solution at the same coordinate of the following integrodifferential equation, namely, the DM-NLSE:

$$
\begin{aligned}
& \frac{\partial \widetilde{U}(z, \omega)}{\partial z}=-\frac{j \omega^{2} \widetilde{U}(z, \omega)}{2 L_{D}}-j \iint \widetilde{R}\left(\omega_{1} \omega_{2}\right) \\
& \times \widetilde{U}\left(z, \omega+\omega_{1}\right) \widetilde{U}\left(z, \omega+\omega_{2}\right) \widetilde{U}^{*}\left(z, \omega+\omega_{1}+\omega_{2}\right) \frac{d \omega_{1}}{2 \pi} \frac{d \omega_{2}}{2 \pi}
\end{aligned}
$$

where $L_{D} \triangleq T^{2} /\left\langle\beta_{2}\right\rangle$ is the average dispersion length and $\widetilde{R}\left(\omega_{1} \omega_{2}\right)$ is the kernel of the map

$$
\widetilde{R}\left(\omega_{1} \omega_{2}\right)=\left\langle\frac{f(z) \exp \left(j \int_{0}^{z} \frac{\omega_{1} \omega_{2}}{L_{\Delta}(x)} d x\right)}{L_{\mathrm{n}}(z)}\right\rangle
$$

being $L_{\Delta}(z) \triangleq T^{2} / \Delta \beta_{2}(z)$ the differential dispersion length. The same DM-NLSE can be obtained by applying to (2) the method of averaging [27], as done in [6]. Note that in the above DM-NLSE, the propagation variable $z$ is not normalized, differently from [2].

Unfortunately, the numerical evaluation of the DM-NLSE is in general slower than that of the NLSE [21]; hence it is not useful to run practical simulations. However, we will show that it allows one to get deep insight into the scaling laws that rule DM systems, as well as to derive several interesting optimization rules.

In the time domain $\tau$, the DM-NLSE takes the following form [2]:

$$
\begin{array}{r}
\frac{\partial U(z, \tau)}{\partial z}=\frac{j}{2 L_{D}} \frac{\partial^{2} U(z, \tau)}{\partial \tau^{2}}-j \iint R\left(\tau_{1} \tau_{2}\right) U\left(z, \tau+\tau_{1}\right) \\
\times U\left(z, \tau+\tau_{2}\right) U^{*}\left(z, \tau+\tau_{1}+\tau_{2}\right) d \tau_{1} d \tau_{2}
\end{array}
$$

and the two-dimensional inverse Fourier transform of the kernel (4) is

$$
R\left(\tau_{1} \tau_{2}\right)=\left\langle\frac{f(z) \exp \left(-j \tau_{1} \tau_{2} / \int_{0}^{z} \frac{1}{L_{\Delta}(x)} d x\right)}{2 \pi L_{\mathrm{n}}(z) \int_{0}^{z} \frac{1}{\left|L_{\Delta}(x)\right|} d x}\right\rangle .
$$

Equations (4) and (6) clarify that the kernel is a function of the products $\omega_{1} \omega_{2}$ or $\tau_{1} \tau_{2}$ only, respectively, in the frequency/time 
domains. At this stage, it is useful to introduce the nonlinear phase cumulated by a constant signal with power $P$ as

$$
\Phi_{\mathrm{NL}}(z)=\int_{0}^{z} \frac{f(x)}{L_{\mathrm{n}}(x)} d x \triangleq \frac{z}{L_{\mathrm{NL}}}
$$

which implicitly defines the span-averaged nonlinear length $L_{\mathrm{NL}}$. Note that $\Phi_{\mathrm{NL}}=\widetilde{R}(0) z$.

Throughout this paper, we will focus on a typical terrestrial link, with span length $L$ much longer than the effective length of transmission fiber [22] and negligible nonlinearity in the compensating fibers, yielding the following simple expression of the kernel (4) [20]:

$$
\lim _{L \rightarrow \infty} \widetilde{R}\left(\omega_{1} \omega_{2}\right) z=\frac{\Phi_{\mathrm{NL}}}{1+j S \omega_{1} \omega_{2}}
$$

where $S \triangleq-L_{A} / L_{\Delta}$ is the map strength of the terrestrial link, $L_{A} \triangleq 1 / \alpha$ is the attenuation length of the transmission fiber, and the differential dispersion length $L_{\Delta}$ refers to its $z$-independent value along the transmission fiber. The map strength slightly differs from the parameter $-\beta_{2} R^{2} L_{\mathrm{eff}}$ introduced in [8] (where $L_{\text {eff }}=L<f>\simeq L_{A}$ for long spans $L$ ), which depends on the transmission fiber dispersion instead of the differential dispersion. For general nonsymmetric DM-links, it is shown in [20] that, through the introduction of an equivalent strength, the kernel $\widetilde{R}$ can still be approximated as in (8).

From the form of the terrestrial kernel (8) and the DM-NLSE (3), one easily deduces that system performance for typical terrestrial systems, i.e., nonsoliton systems in which average GVD and nonlinear effects weakly interact, only depends on the following dimensionless parameters: 1$)$ the nonlinear cumulated phase $\Phi_{\mathrm{NL}}(z)=z / L_{\mathrm{NL}}, 2$ ) the map strength $S$, and 3 ) the (normalized) inline cumulated dispersion $\xi_{\text {in }}(z) \triangleq z / L_{D}$. The connection of these parameters with the standard fiber parameters is summarized for convenience in Appendix I. Note that, although in this paper we are mostly interested in small $\Phi_{\mathrm{NL}}$ DM terrestrial systems, the validity of the DM-NLSE also extends to large $\Phi_{\mathrm{NL}}$ DM-soliton systems [1]-[3].

Clearly, also pre- and postcompensation play an important role, but they do not explicitly appear in (3). We will denote with $\xi_{\text {pre }}$ and $\xi_{\text {post }}$ their normalized cumulated dispersions, as defined in Appendix I. We also define $\xi_{\text {tot }} \triangleq \xi_{\text {pre }}+\xi_{\text {in }}+\xi_{\text {post }}$ as the total (also called residual [17], [25]) dispersion of the link. A summary of such normalized dispersions appears in the inset of Fig. 1. Optimization criteria for pre- and postcompensation will be considered in Section IV.

In the time domain, the terrestrial kernel (8) has the following expression:

$$
\lim _{L \rightarrow \infty} R\left(\tau_{1} \tau_{2}\right) z=\frac{\Phi_{\mathrm{NL}}}{\pi|S|} K_{0}\left(2 \sqrt{\frac{\tau_{1} \tau_{2}}{j S}}\right)
$$

where $K_{0}(x)$ is the modified Bessel function of the second kind of order zero. Note from (9) that reversing the sign of $S$ corresponds to conjugating the kernel, since the Taylor series expansion of $K_{0}(z)$ has real coefficients. By exploiting the asymptotic

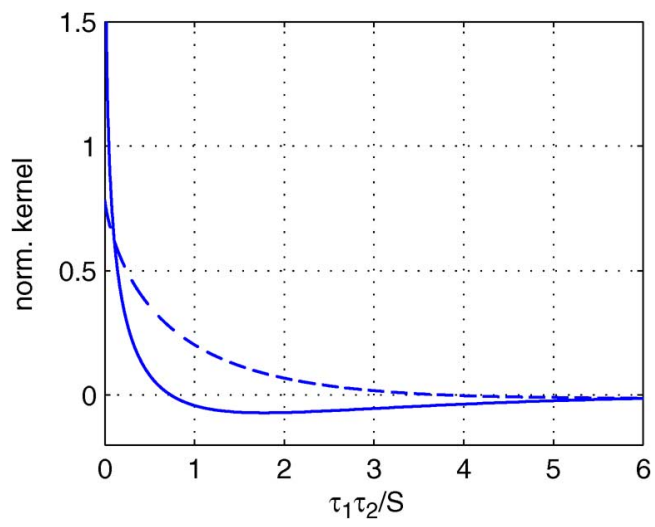

Fig. 2. Real (solid) and imaginary (dashed) parts of $\left(\pi|S| / \Phi_{\mathrm{NL}}\right) R\left(\tau_{1} \tau_{2} / S\right) z$ versus $\tau_{1} \tau_{2} / S$.

behavior of $K_{0}(x)$, one gets the following useful asymptotic expansions:

$$
R z \cong \begin{cases}-\frac{\Phi_{\mathrm{NL}}}{2 \pi|S|}\left(\log 2-j \frac{\pi}{2}+\log \left(\frac{2 \tau_{1} \tau_{2}}{S}\right)\right) & \frac{\tau_{1} \tau_{2}}{S} \rightarrow 0 \\ \frac{\Phi_{\mathrm{NL}}}{\sqrt{2 \pi|S|}} \frac{\exp \left(-(1-j) \sqrt{\frac{2 \tau_{1} \tau_{2}}{S}}\right)}{\left(\frac{2 \tau_{1} \tau_{2}}{S}\right)^{1 / 4}} & \frac{\tau_{1} \tau_{2}}{S} \rightarrow \pm \infty\end{cases}
$$

which show that $|R z|$ approaches infinity as $\tau_{1} \tau_{2} / S \rightarrow 0$ and that $|R z|$ behaves like a Dirac delta for $S \rightarrow 0$. In Fig. 2, we plot the real and imaginary part of $R z$ (normalized to $\Phi_{\mathrm{NL}} / \pi|S|$ ) versus $\tau_{1} \tau_{2} / S$ in order to give a universal representation for any $S$ and $\Phi_{\mathrm{NL}}$. We note from the figure that the kernel is asymptotically close to zero starting from $\tau_{1} \tau_{2} / S>5$. Even if such a region is unbounded in the $\tau_{1}, \tau_{2}$ plane, the contribution of the kernel to the DM-NLSE (5) is significant only in a limited region around $\tau_{1}=\tau_{2}=0$. A numerical computation (not shown here) shows in fact that the integral $A_{R}(\tau)=$ $\left(\pi|S| / \Phi_{\mathrm{NL}}\right) \int_{-\tau}^{\tau} \int_{-\tau}^{\tau} R\left(\tau_{1} \tau_{2}\right) z d \tau_{1} d \tau_{2}$ saturates at $\tau \cong \sqrt{5 S}$, a fact that reveals the existence of a finite memory of the DM-link, which will be further discussed in Section VI-B.

\section{USE OF DM-NLSE IN LIMITED-SPAN DM TERRESTRIAL SYSTEMS}

Since the main assumption of the multiple scales is that, over the fast scale, the nonlinearity is sufficiently small [21], we tested the reliability of the DM-NLSE in practical terrestrial systems by varying the nonlinear phase per span, which was achieved by fixing the total nonlinear phase and then increasing the number of spans $N_{s}$. For a finite total nonlinear phase, the solution of the DM-NLSE then coincides with the solution of the NLSE in the limit of an infinite number of spans.

As a case study, we analyzed an $R=10 \mathrm{~Gb} / \mathrm{s}$ single-channel NRZ-OOK terrestrial system having transmission fiber dispersion of $D_{\mathrm{T}}=8 \mathrm{ps} / \mathrm{nm} / \mathrm{km}$ (strength $S=0.022$ ) with full in-line compensation $\left(\xi_{\text {in }}=0\right)$, optimized precompensation, and zero residual dispersion $\left(\xi_{\text {tot }}=0\right)$.

We first evaluated the DM-NLSE solution $U_{\mathrm{DM}}(\tau)$ with the algorithm detailed in [21] for some values of the nonlinear phase in the range $0.1 \pi \div 2 \pi$. In all cases we found an excellent agreement between $U_{\mathrm{DM}}(\tau)$ and direct SSFM solution of the NLSE 

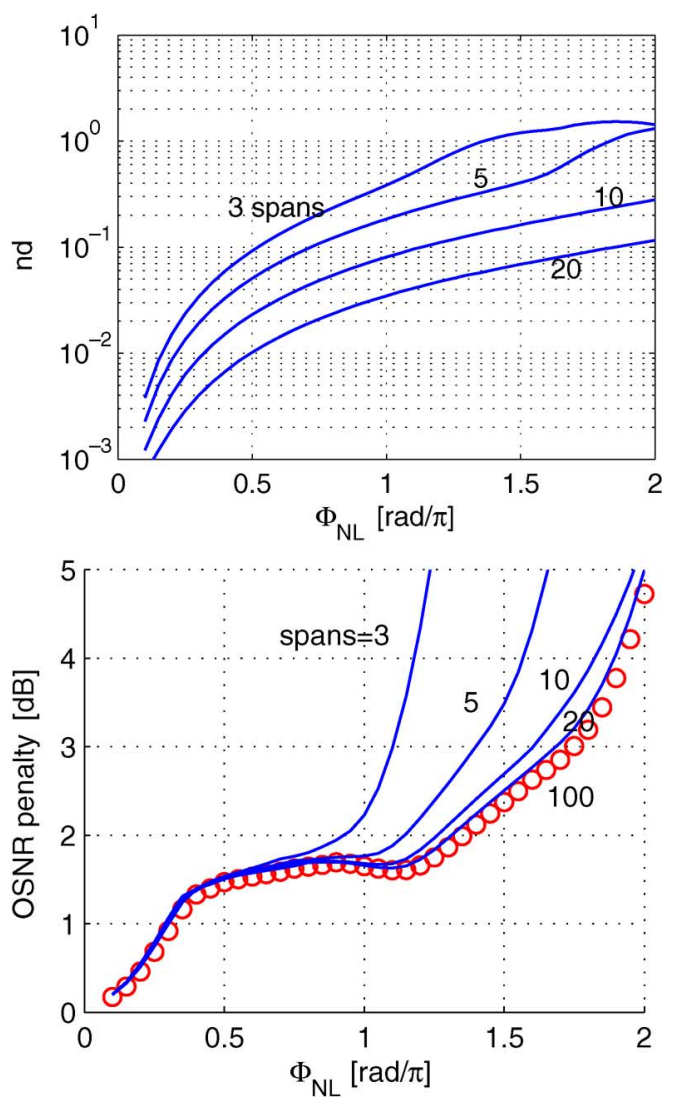

Fig. 3. (Top) $n d$ versus $\Phi_{\mathrm{NL}}$. (Bottom) OSNR penalty at BER $=10^{-5}$ versus $\Phi_{\mathrm{NL}}$. Fully compensated $D_{\mathrm{T}}=8 \mathrm{ps} / \mathrm{nm} / \mathrm{km}$ system at $R=10 \mathrm{~Gb} / \mathrm{s}(S=$ 0.022 ).

with $N_{s}=100$ spans, $U_{100}(\tau)$, as quantified by the normalized deviation [28]

$$
n d=\frac{\left\|U_{\mathrm{DM}}(\tau)-U_{100}(\tau)\right\|}{\left\|U_{100}(\tau)\right\|}
$$

where $\|U\| \triangleq\left(\int|U|^{2} d \tau\right)^{1 / 2}$. In all cases, we found $n d<$ $10^{-3}$, which amounts to a difference between the two waveforms hardly perceivable by eye. In the following, we thus used the 100-span SSFM solution as the "reference" DM-NLSE solution because of its significant savings in computation time.

We next varied the number of spans in the range $N_{s}=1 \div$ 30 and the $\Phi_{\mathrm{NL}}$ cumulated by the signal average power in the range $0.1 \pi \div 2 \pi$ with fine steps of $0.05 \pi$. After propagation on $N_{s}=k$ spans, we measured the received electric field $U_{k}(\tau)$ by solving the NLSE with the SSFM algorithm. The SSFM was applied using a variable step-size with a maximum nonlinear phase cumulated within each step equal to $5 \cdot 10^{-5} \mathrm{rad}$ and a maximum step-size of $5 \mathrm{~km}$. The collection of received electric fields was then compared with the DM-NLSE case $\left(N_{s}=100\right)$, and the distance $n d(k)$ between $U_{k}(\tau)$ and $U_{100}(\tau)$ was recorded, using $U_{k}(\tau)$ in place of $U_{\mathrm{DM}}(\tau)$ in (10). Fig. 3 (top) shows $n d(k)$ versus the nonlinear phase $\Phi_{\mathrm{NL}}$ for an increasing number of spans $k$. From the figure, we observe that, for a given $\Phi_{\mathrm{NL}}$, $n d(k)$ decreases for increasing $k$, as expected.

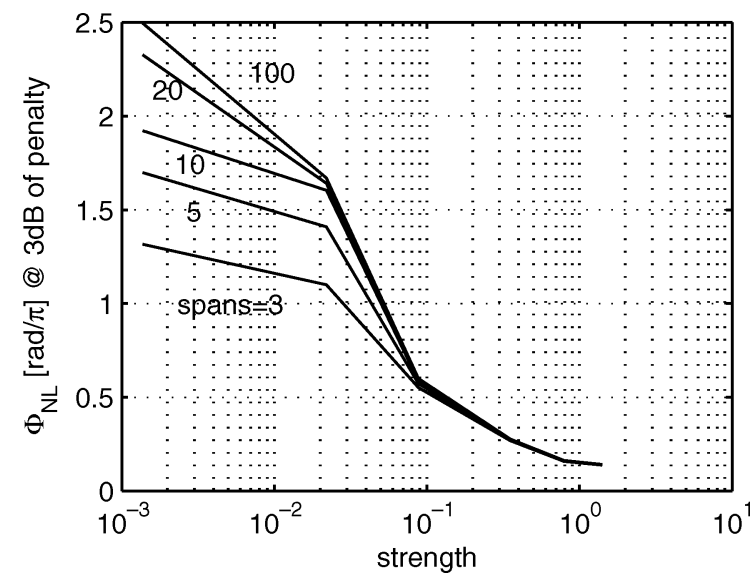

Fig. 4. $\Phi_{\mathrm{NL}}$ that yields $3 \mathrm{~dB}$ of OSNR penalty versus map strength. Fully compensated system, optimized precompensation, zero residual dispersion.

The $n d$ distance is one measure of waveform similarity but does not provide information about the "closeness" of system performance, as expressed, for instance, by the OSNR penalty. Hence, while measuring the $n d$, we also monitored the OSNR penalty at BER $=10^{-5}$, both using $U_{100}$ and $U_{k}$. The OSNR penalty was evaluated from the exact BER using the Karhunen-Loève (KL) method [29]. In Fig. 3 (bottom), we report the OSNR penalty versus nonlinear phase $\Phi_{\mathrm{NL}}$ by varying the number of spans. In agreement with Fig. 3 (top), we note that the OSNR penalty gets closer to that of the 100 -span case over a wider $\Phi_{\mathrm{NL}}$ range for larger $k$, and that systems with smaller number of spans have a larger penalty, since the nonlinear phase per span becomes so large as to violate the main assumption of the DM-NLSE, i.e., only the differential GVD operates along the fast scale. By comparing the top and bottom of Fig. 3, we also note that a relatively large value of $n d<10^{-1}$ guarantees a proper evaluation of OSNR penalty at BER $=10^{-5}$ using the DM-NLSE (at smaller $\mathrm{BER}$, the required $n d$ is much smaller, but terrestrial systems are usually employed with forward error correcting codes, so that BER values smaller than $10^{-5}$ are not of interest). For instance, we see that at strength $S=0.022(R=10 \mathrm{~Gb} / \mathrm{s}$ on $D_{\mathrm{T}}=8 \mathrm{ps} / \mathrm{nm} / \mathrm{km}$ ), a three-span fully compensated system can be approximated by the DM-NLSE provided that the nonlinear phase is $\Phi_{\mathrm{NL}}<0.5 \pi[\mathrm{rad}](0.5 \mathrm{rad} / \mathrm{span})$, a ten-span system for $\Phi_{\mathrm{NL}}<\pi$ [rad] $(0.3 \mathrm{rad} / \mathrm{span})$, and a 20 -span system for $\Phi_{\mathrm{NL}}<1.8 \pi[\mathrm{rad}](0.28 \mathrm{rad} / \mathrm{span})$.

By slicing Fig. 3 (bottom) at a level of $3 \mathrm{~dB}$, one gets, for each specific span number, the NLT value $\Phi_{\mathrm{NL}}$ that yields 3 $\mathrm{dB}$ of OSNR penalty atBER $=10^{-5}$ at the specific strength $S=0.022$. We repeated the measurement of the NLT for other strength values while keeping the remaining system parameters unchanged, thus obtaining the curves shown in Fig. 4. We note that the accuracy of the DM-NLSE (curve labeled 100) is larger for increasing map strength $S$, since a larger strength implies a faster variation of the GVD within each span with a more effective averaging operation, as required by the DM-NLSE.

Finally, Fig. 5 shows the $\Phi_{\mathrm{NL}}$ that yields $n d=10^{-2}$ versus inline cumulated dispersion. The system was an $N_{s}=30$ spans with $R=10 \mathrm{~Gb} / \mathrm{s}$. The figure is provided to show that zero 


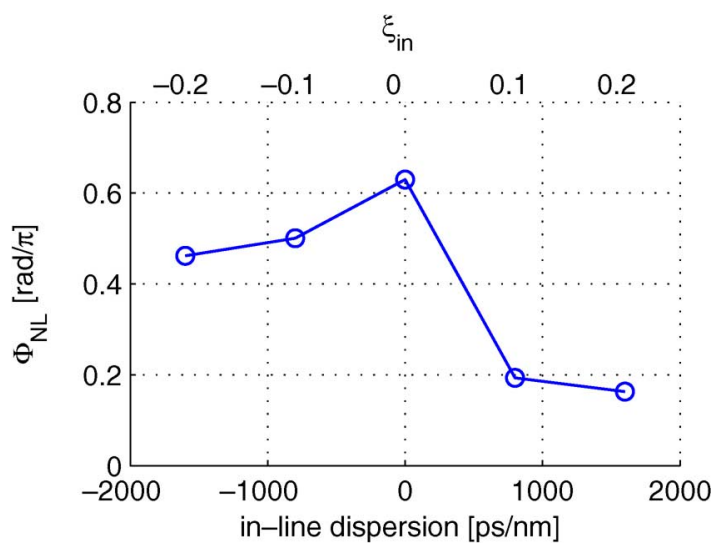

Fig. 5. $\Phi_{\mathrm{NL}}$ at $n d=10^{-2}$ versus inline dispersion. Thirty-span system at $R=10 \mathrm{~Gb} / \mathrm{s} . D_{\mathrm{T}}=8 \mathrm{ps} / \mathrm{nm} / \mathrm{km}$.

inline dispersion is a benign case for DM-NLSE accuracy and that the accuracy is worse at positive inline dispersions.

\section{LARge-Signal Perturbative AnAlysis}

We saw in Section II that the three parameters $\Phi_{\mathrm{NL}}, S, \xi_{\text {in }}$ are the only free parameters explicitly appearing in the DM-NLSE for a terrestrial link. We will now discuss the role of the preand postcompensation $\xi_{\text {pre }}$ and $\xi_{\text {post }}$, respectively. For their relation with standard units, see Appendix I. A summary of such normalized dispersions appears in the inset of Fig. 1.

The DM-NLSE cannot be solved exactly except in a few cases, e.g., when $S \rightarrow 0$. Thus, in order to gain physical insight about the effect of pre- and postcompensation on the DM-NLSE (3), we search for an enhanced regular perturbation solution in $1 / L_{\mathrm{NL}}$ [27], [30]. The initial condition is $\widetilde{U}(0, \omega) \triangleq \exp \left(j\left(\omega^{2} / 2\right) \xi_{\text {pre }}\right) \widetilde{U}_{i}(\omega)$, with $\widetilde{U}_{i}(\omega)$ being the unchirped field transmitted by the laser source and $\xi_{\text {pre }}$ the precompensation. We start with a change of variable that removes the average nonlinear cumulated phase and the inline cumulated GVD

$$
\widetilde{U}(z, \omega) \triangleq \widetilde{E}(z, \omega) e^{-j \frac{\omega^{2}}{2} \frac{z}{L_{D}}} e^{-j \Phi_{\mathrm{NL}}(z)}
$$

and then expand the new field $\widetilde{E}(z, \omega)=\widetilde{E}_{0}+\widetilde{E}_{1} / L_{\mathrm{NL}}+$ $\widetilde{E}_{2} / L_{\mathrm{NL}}^{2} \ldots$ in series of $1 / L_{\mathrm{NL}}$. Using such an expansion in (11), and the result into (3), it is possible to solve iteratively (3) by equating equal powers in $1 / L_{\mathrm{NL}}$ [30]. If we stop the series to first power in $1 / L_{\mathrm{NL}}$, we obtain the following first-order perturbative solution of (3)

$$
\widetilde{U}(z, \omega) \cong e^{-j \frac{\omega^{2}}{2} \frac{z}{L_{D}}-j \Phi_{\mathrm{NL}}}\left(\widetilde{E}_{0}(\omega)+\frac{1}{L_{\mathrm{NL}}} \widetilde{E}_{1}(\omega)\right)
$$

with $\widetilde{E}_{0}=\widetilde{U}(0, \omega)$ and where the first-order perturbative term is

$$
\begin{aligned}
& \frac{\widetilde{E}_{1}(\omega)}{L_{\mathrm{NL}}}=j \Phi_{\mathrm{NL}} \widetilde{U}(0, \omega)-j \iint \widetilde{R}_{S}\left(\omega_{1} \omega_{2}\right) \\
& \times \widetilde{U}\left(0, \omega+\omega_{1}\right) \widetilde{U}\left(0, \omega+\omega_{2}\right) \widetilde{U}^{*}\left(0, \omega+\omega_{1}+\omega_{2}\right) \frac{d \omega_{1}}{2 \pi} \frac{d \omega_{2}}{2 \pi}
\end{aligned}
$$

which has now a modified kernel

$$
\begin{aligned}
\widetilde{R}_{S}\left(\omega_{1} \omega_{2}\right) & =\widetilde{R}\left(\omega_{1} \omega_{2}\right) \int_{0}^{z} e^{+j \omega_{1} \omega_{2} \frac{x}{L_{D}}} d x \\
& =\Phi_{\mathrm{NL}} \frac{e^{+\frac{j \omega_{1} \omega_{2} z}{2 L_{D}}}}{1+j S \omega_{1} \omega_{2}} \frac{\sin \left(\frac{z}{2 L_{D}} \omega_{1} \omega_{2}\right)}{\frac{z}{2 L_{D}} \omega_{1} \omega_{2}} .
\end{aligned}
$$

Once an expression for the field $\widetilde{U}(z, \omega)$ at the output of the optical link is found, the received field after postcompensation can be written as $\widetilde{U}_{o}(\omega)=\exp \left(j\left(\omega^{2} / 2\right) \xi_{\text {post }}\right) \widetilde{U}(z, \omega)$, with $\xi_{\text {post }}$ being the normalized postcompensation. Using (12) and (13), we get

$$
\widetilde{U}_{o}(\omega)=e^{j \frac{\omega^{2}}{2} \xi_{\mathrm{tot}}-j \Phi_{\mathrm{NL}}}\left(\widetilde{U}_{i}(\omega)+\Phi_{\mathrm{NL}} \widetilde{U}_{N}(\omega)\right)
$$

where

$$
\begin{array}{r}
\widetilde{U}_{N}(\omega)=+j \widetilde{U}_{i}(\omega)-j \iint \widetilde{\eta}\left(\omega_{1} \omega_{2}\right) \widetilde{U}_{i}\left(\omega+\omega_{1}\right) \widetilde{U}_{i}\left(\omega+\omega_{2}\right) \\
\times \widetilde{U}_{i}^{*}\left(\omega+\omega_{1}+\omega_{2}\right) \frac{d \omega_{1}}{2 \pi} \frac{d \omega_{2}}{2 \pi}
\end{array}
$$

and where the kernel $\widetilde{\eta}$ equals

$$
\widetilde{\eta}\left(\omega_{1} \omega_{2}\right)=\frac{e^{-j \omega_{1} \omega_{2}\left(\xi_{\text {pre }}+\xi_{\text {in }} / 2\right)}}{1+j S \omega_{1} \omega_{2}} \frac{\sin \left(\xi_{\text {in }} \omega_{1} \omega_{2} / 2\right)}{\xi_{\text {in }} \omega_{1} \omega_{2} / 2} .
$$

It is worth noting from (15) that even with a perfect compensation of the GVD (i.e., $\xi_{\text {tot }}=0$ ), some amount of distortion remains from the perturbative term $\widetilde{U}_{N}(\omega)$. We now ask if, in such a condition of zero residual GVD, a prefiber exists that minimizes the distortion on the received signal. For this purpose, we evaluate the received power using the inverse Fourier transform of (15) with $\xi_{\text {tot }}=0$

$$
\left|U_{o}(\tau)\right|^{2}=\left|U_{i}+\Phi_{\mathrm{NL}} U_{N}\right|^{2} \cong\left|U_{i}\right|^{2}+2 \Phi_{\mathrm{NL}} \Re \mathrm{e}\left\{U_{i}^{*} U_{N}\right\}
$$

where $\Re \mathrm{e}\{x\}$ indicates the real part of the complex number $x$ and the last approximation is consistent with keeping first-order terms in $1 / L_{\mathrm{NL}}$ only. Assuming that $U_{i}(\tau)$ is a real chirpless signal, the received power is equal to the transmitted one if $U_{N}(\tau)$ is imaginary. This happens if $\widetilde{U}_{N}(\omega)=-\widetilde{U}_{N}^{*}(-\omega)$, a condition that from (16) can be met with $\Im \mathrm{m}\left\{\widetilde{\eta}\left(\omega_{1} \omega_{2}\right)\right\}=0$, with $\Im$ m being the imaginary part. From (17), we get a real kernel in the frequency domain by imposing the condition

$$
\xi_{\text {pre }} \omega_{1} \omega_{2}=-\arctan \left(S \omega_{1} \omega_{2}\right)-\frac{\xi_{\text {in }}}{2} \omega_{1} \omega_{2} .
$$

Condition (18) cannot be met by all pairs $\left(\omega_{1}, \omega_{2}\right)$. The best that can be done is equalizing the first-order Taylor expansion of the $\arctan$ term, i.e., $\arctan \left(S \omega_{1} \omega_{2}\right) \cong S \omega_{1} \omega_{2}$, obtaining an optimal precompensation equal to

$$
\left.\xi_{\text {pre }}\right|_{o p t}=-S-\frac{\xi_{\text {in }}}{2} .
$$

Equation (19) is the straight-line rule (SLR) mentioned in the introduction for a terrestrial DM system and supports the empirical rule found in [15] and explained in [16] with a simple phenomenological model. It also has the same functional 
behavior as the rule proposed in [13] based on minimization of intrachannel cross-phase modulation, except for a $\log (2)$ factor multiplying $S$ in the SLR in [13], which is not needed to best match the optimal precompensation for NRZ-OOK found by both simulations and experiments. Surprisingly, it is needed instead for the optimal precompensation of NRZ differential phase-shift keying (DPSK) [31]. The connection between the SLR and Killey's formula can be understood from the elegant work of Wey [32], where the same manipulations of the NLSE and the perturbative method are applied without the span-averaging of the DM-NLSE. Wey arrives at a kernel [32], (5) whose specialization to a terrestrial DM link [32], Figs. 1 and 2 coincides with our kernel expression (17) in the limit of infinitely many spans with infinitesimal residual dispersion per span. Wey shows that, by considering $\omega_{1} \omega_{2} \triangleq \zeta$ as a single "frequency" variable, the inverse Fourier transform of $\widetilde{\eta}(\zeta)$ is a function $J(c)$ whose physical interpretation is a power-weighted dispersion distribution $[c(z)$ representing the cumulated dispersion up to coordinate $z$ ] and in probability theory it would be called a probability density function (pdf). It is simple to show that our SLR corresponds to choosing $\xi_{\text {pre }}$ so as to set the average value of such a pdf to zero, while Killey's formula (for a single DM span) corresponds to setting the median of $J(c)$ to zero.

We will numerically verify the accuracy of the SLR (19) in Section VI-A. Such optimal precompensation was derived in the assumption of zero residual GVD and amounts to creating an optical link as close as possible to one with only self-phase modulation (SPM), for which it is exactly $\widetilde{U}_{N}(\omega)=-\widetilde{U}_{N}^{*}(-\omega)$. Therefore, the received pulse intensity is almost distortionless in the limit of the perturbative approach, i.e., for small $\Phi_{\mathrm{NL}}$. However, with only SPM, one can improve OOK performance by adding a proper postcompensation [33] by having the chirp induced by SPM collaborate with that of the postfiber to compress the pulses. Hence, to the postdispersion derived with the perturbation approach, i.e., $\xi_{\mathrm{p} 1}=S-\xi_{\text {in }} / 2$, one should add the optimal dispersion found in [33], which translates into our dimensionless units as

$$
\xi_{\mathrm{p} 2}=\frac{\sqrt{2}}{8} \frac{\operatorname{sign}(S) \Phi_{\mathrm{NL}}}{1+\frac{4}{3 \sqrt{3}} \Phi_{\mathrm{NL}}^{2}}
$$

where $\Phi_{\mathrm{NL}}$ is the peak nonlinear phase cumulated by the signal. ${ }^{2}$ The optimal postdispersion is then $\xi_{\text {post }}=\xi_{\mathrm{p} 1}+\xi_{\mathrm{p} 2}$. Note that (20) justifies the strength-independent linear growth of the optimal residual dispersion with $\Phi_{\mathrm{NL}}$ found in DM-optimized $40 \mathrm{~Gb} / \mathrm{s}$ systems [17], in which the allowed nonlinear phases are small and correspond to the linear initial portion of the curve in (20).

The perturbative approach clearly works at small values of $\Phi_{\mathrm{NL}}$. In order to measure its accuracy, we tested in separate simulations, not fully reported here, the normalized deviation $n d$ between the perturbative solution (12) and the NLSE solution for a fully compensated 20 -span terrestrial system working in absence of pre- and postcompensating fibers. We observed a

\footnotetext{
${ }^{2}$ Since (20) is based on the assumption of Gaussian pulses, we referred the normalized dispersion $\xi_{\mathrm{p} 2}$ to a reference time $T$ equal to twice the $1 / e$ pulse-
} duration.

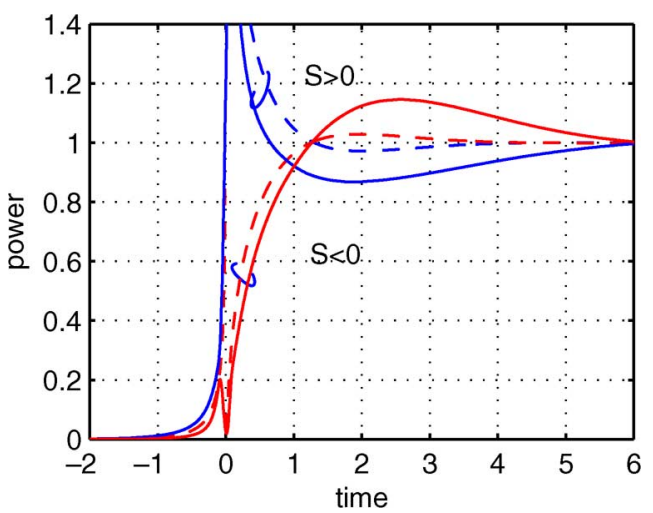

Fig. 6. Step response (normalized to the peak power) of a DM link with $S=$ $2.2, \xi_{\text {in }}=\xi_{\text {pre }}=\xi_{\text {post }}=0$. (Solid lines) SSFM simulations; (dashed lines) (21).

value $n d=0.1$ at $\Phi_{\mathrm{NL}} \cong 0.3 \pi$, almost independently of the map strength value over the range $S=0.02 \div 2$. More results on the validity range of the perturbative approach can be found in [30].

\section{A. Large-Signal Step Response of the DM-Link}

A perturbative approach applied to the NLSE has been successfully used to derive the energy growth of ghost pulses in strongly dispersion managed systems having signals with Gaussian supporting pulses [3], [14]. Here we approach the problem by analyzing the step response of the optical DM-link, which describes the case of ideal NRZ pulses and transmitted bit sequence ...000111 ... To this aim, we use the perturbative solution (12) excited with $U(0, \tau)=u(\tau)$, with $u(\tau)$ being the step function, i.e., $u(\tau)=1, \tau>0$, and zero otherwise. This choice implies that the reference power $P$ in (7) is the signal peak power and, consequently, $\Phi_{\mathrm{NL}}$ here is the cumulative peak nonlinear phase. At full inline compensation, $\xi_{\text {in }}=0$, one can obtain a closed-form of the double integral in (16) with a step input

$$
U(z, \tau)=e^{-j \Phi_{\mathrm{NL}}}\left(u(\tau)+\operatorname{sgn}[S] \frac{\Phi_{\mathrm{NL}}}{\pi} K_{0}\left(2 \frac{|\tau|}{\sqrt{j S}}\right)\right)
$$

which is the exact first-order perturbative solution (12) for $\tau<0$ and an approximation of it for $\tau>0$. Fig. 6 shows the step response for a system having $S=2.2, \xi_{\text {in }}=0$, and $\Phi_{\mathrm{NL}}=$ $0.2 \pi$. The dashed lines represent (21), while SSFM simulations are in solid lines. We note that (21) well reproduces the overflow of energy on the space bits close to the step, while for $\tau>0$, it reasonably captures the qualitative behavior of the distortion.

Such a perturbative solution of the step-response can be used, for instance, to evaluate the energy that falls into the first space before the step, within $-1<\tau<0$, which is the energy captured by an OBPF integrator, i.e., the matched filter for ideal NRZ pulses, and thus gives the current on the most penalized space in the sequence ...000111... Such an energy is $E_{0}=$ $|\mathcal{E}|^{2}$, where the electric field $\mathcal{E}=\int_{-1}^{0} U(z, \tau) d \tau$ has expression

$$
\mathcal{E}=\frac{\operatorname{sgn}[S] \Phi_{\mathrm{NL}}}{2 \pi} \sqrt{j S}\left\{\frac{\pi}{2}-\int_{0}^{\infty} \operatorname{sech}(y) e^{-\frac{2 \cosh y}{\sqrt{j S}}} d y\right\} .
$$




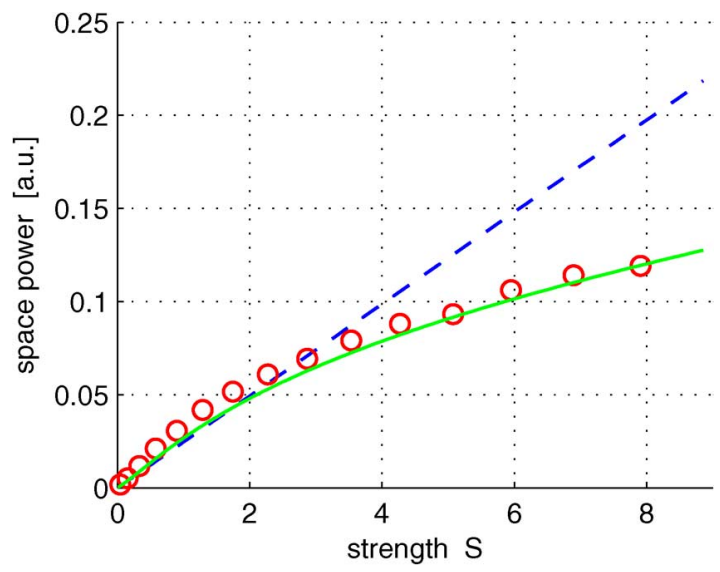

Fig. 7. Energy of the first space before marks of NRZ sequence . . . 000111 . . versus $S$ in a DM link with $\Phi_{\mathrm{NL}}=0.2 \pi, \xi_{\text {in }}=\xi_{\text {pre }}=\xi_{\text {post }}=0$. (Circles) SSFM simulations; (solid and dashed line) (23).

In (22), we made use of the integral representation of the Bessel function, i.e., $K_{0}(x)=\int_{0}^{\infty} \exp (-x \cosh (y)) d y$, and used $\int_{0}^{\infty} \operatorname{sech}(\tau) d \tau=\pi / 2$. The last integral in (22) becomes relevant only at very large $S$, and a good approximation can be obtained by using the Laplace method [34]

$$
\mathcal{E} \cong \frac{\operatorname{sgn}[S] \Phi_{\mathrm{NL}}}{2 \pi} \sqrt{j S}\left\{\frac{\pi}{2}-\frac{1}{2} \sqrt{\pi \sqrt{j S}} e^{-\frac{2}{\sqrt{j S}}}\right\} .
$$

In Fig. 7 we plot $E_{0}$ versus $S$ for a $20 \times 100 \mathrm{~km}$ fully compensated system with $\Phi_{\mathrm{NL}}=0.2 \pi$. The circles refer to SSFM simulations applied to a step signal, while the solid line refers to the approximation (23). The dashed line refers to (22) without the last integral, which corresponds to integrating the space energy from $-\infty$ to zero. Under this assumption, the main conclusion is that $E_{0}$ scales as $\Phi_{\mathrm{NL}}^{2}|S|$. Such an approximation is unreliable at large strengths where the collapse of energy into the space exceeds $\tau=-1$.

Note that the growth of interpulse four-wave mixing with increasing map strength shown in Fig. 7 is consistent with the results in [3, Fig. 7 (bottom)]. Also, using a totally different approach, [35] reached similar qualitative conclusions regarding the generation of ghost pulses preceding a long string of marks in an RZ-DM system (the asymmetric case in [35]).

\section{SMAll-Signal ANALYSis}

In this section, we investigate a small-signal solution of the DM-NLSE using linearization. To this aim, we write the normalized electric field as the sum of a continuous wave $(\mathrm{CW})$ and a zero-average small perturbative term $a(z, \tau)$

$$
U(z, \tau)=(1+a(z, \tau)) e^{-j \Phi_{\mathrm{NL}}(z)}
$$

where $\Phi_{\mathrm{NL}}(z)$ is the nonlinear phase cumulated by the CW up to $z$ and $a(z, \tau) \triangleq a_{p}(z, \tau)+j a_{q}(z, \tau)$, with $a_{p}$ and $a_{q}$ being the real in-phase and quadrature components of $a$ in a reference system rotated by $\Phi_{\mathrm{NL}}$. Note that $P$ in (7) is the signal average power. Substituting the ansatz (24) in (3) and using the assumption $|a| \ll 1$, the DM-NLSE can be linearized, yielding a linear propagation equation $\mathbf{a}(z, \omega)=\mathbf{T}(z, \omega) \cdot \mathbf{a}(0, \omega)$ for the column vector $\mathbf{a}(z, \omega)=\left[\tilde{a}_{p}, \tilde{a}_{q}\right]^{T}$ of the Fourier transforms $\left(\tilde{a}_{p}, \tilde{a}_{q}\right)$ of $\left(a_{p}, a_{q}\right)$, where symbol ${ }^{T}$ indicates transposition. As shown in [20] through a direct derivation of the linearized DM-NLSE, the DM link matrix $\mathbf{T}$ is

$$
\mathbf{T} \triangleq e^{\mathbf{M}(\boldsymbol{\omega}) z}=\cosh (k z) \mathbf{I}+\frac{\sinh (k z)}{k z} \mathbf{M} z
$$

with $\mathbf{I}$ being the $2 \times 2$ identity matrix and

$$
\mathbf{M} z=\left[\begin{array}{cc}
\widetilde{R}_{I}\left(\omega^{2}\right) & \Phi_{\mathrm{NL}}-\frac{\omega^{2}}{2} \xi_{\text {in }}-\widetilde{R}_{R}\left(\omega^{2}\right) \\
-\Phi_{\mathrm{NL}}+\frac{\omega^{2}}{2} \xi_{\mathrm{in}}-\widetilde{R}_{R}\left(\omega^{2}\right) & -\widetilde{R}_{I}\left(\omega^{2}\right)
\end{array}\right]
$$

where $\widetilde{R}_{R, I}$ are the real/imaginary parts of $\widetilde{R}$, and the $z$-dependence comes through $\Phi_{\mathrm{NL}}$ and $\xi_{\text {in. }}$. The eigenvalues $\pm k z$ of $\mathbf{M} z$ are

$$
k z=\sqrt{\left|\widetilde{R}\left(\omega^{2}\right)\right|^{2}-\left(\Phi_{\mathrm{NL}}-\frac{\omega^{2}}{2} \xi_{\mathrm{in}}\right)^{2}} .
$$

Such a result is valid for any kernel with zero dispersion slope [20]. For the standard terrestrial kernel (8), one has $\widetilde{R}_{R}\left(\omega^{2}\right)=$ $\Phi_{\mathrm{NL}} /\left(1+S^{2} \omega^{4}\right)$, and $\widetilde{R}_{I}\left(\omega^{2}\right)=-\Phi_{\mathrm{NL}} S \omega^{2} /\left(1+S^{2} \omega^{4}\right)$. We now focus on the intensity and phase of the electric field, which, in the small signal assumption, can simply be written in a firstorder Taylor expansion as

$$
\begin{gathered}
\mathcal{P}(z, \tau) \triangleq P|U(z, \tau)|^{2} \cong P+2 P a_{p}(z, \tau) \\
\theta(z, \tau) \triangleq \arg [U(z, \tau)] \cong a_{q}(z, \tau)-\Phi_{\mathrm{NL}} .
\end{gathered}
$$

Moving into the frequency domain, we obtain

$$
\left[\begin{array}{c}
\widetilde{\mathcal{P}}(z, \omega) \\
2 P \widetilde{\theta}(z, \omega)
\end{array}\right] \triangleq \mathbf{v}(z, \omega)=\mathbf{T} \cdot \mathbf{v}(0, \omega)=\mathbf{T}\left[\begin{array}{c}
\widetilde{\mathcal{P}}(0, \omega) \\
2 P \widetilde{\theta}(0, \omega)
\end{array}\right]
$$

where we collected the Fourier transforms of $\mathcal{P}$ and $\theta$ in a column vector $\mathbf{v}(z, \omega)$ and made use of the fact that $\lim _{\omega \rightarrow 0} \mathbf{T} \triangleq \mathbf{T}_{0}=\left[1,0 ;-2 \Phi_{\mathrm{NL}}, 1\right]$. Equation (29) relates the input/output intensity modulation (IM) and phase modulation (PM) of the optical link to the link matrix $\mathbf{T}=\left\{t_{i j}\right\}$, $i, j=1,2$, in which we recognize $t_{11}$ as the transfer function of the IM $\rightarrow \rightarrow$ IM linear system that filters the amplitude of the signal. In the same way, $t_{12}, t_{21}$, and $t_{22}$ play the role of $\mathrm{PM} \rightarrow \mathrm{IM}, \mathrm{IM} \rightarrow \mathrm{PM}$, and $\mathrm{PM} \rightarrow \mathrm{PM}$ filters, respectively.

Note that even if (29) comes from a linearization, $\widetilde{\mathcal{P}}(z, \omega)$ does not violate the conservation of energy, since at $\omega=0$, it is $t_{11}=1$; hence $\int \mathcal{P}(z, \tau) d \tau=\int \mathcal{P}(0, \tau) d \tau$. For instance, the small-signal intensity/phase model (29) at $S=0$ and $\xi_{\text {in }}=0$ has $\mathbf{T}=\mathbf{T}_{0}$ and thus provides the exact large-signal solution: $\mathcal{P}(z, \tau)=|U(0, \tau)|^{2}$ and $\theta(z, \tau)=\arg [U(0, \tau)]-\Phi_{\mathrm{NL}}$. On the contrary, the small-signal in-phase/quadrature field model $\mathbf{a}(z, \omega)=\mathbf{T}(z, \omega) \cdot \mathbf{a}(0, \omega)$ leads to an output intensity $\mathcal{P}(z, \tau)=\left(1+a_{p}(z, \tau)\right)^{2}+a_{q}(z, \tau)^{2}$, which, in general, differs from $|U(0, \tau)|^{2}$. Hence the intensity/phase interpretation (29) of the small signal DM model is preferable, and it gives directly the photodetected current (at unit responsivity of the photodiode). However, whenever an optical filter is present at the receiver, we find it more accurate to convert the phase/intensity to a field $\sqrt{\mathcal{P}(z, \tau)} \exp (j \theta(z, \tau))$, convolve this field with the impulse response of the optical filter, and then take the squared absolute value of the resulting field to 


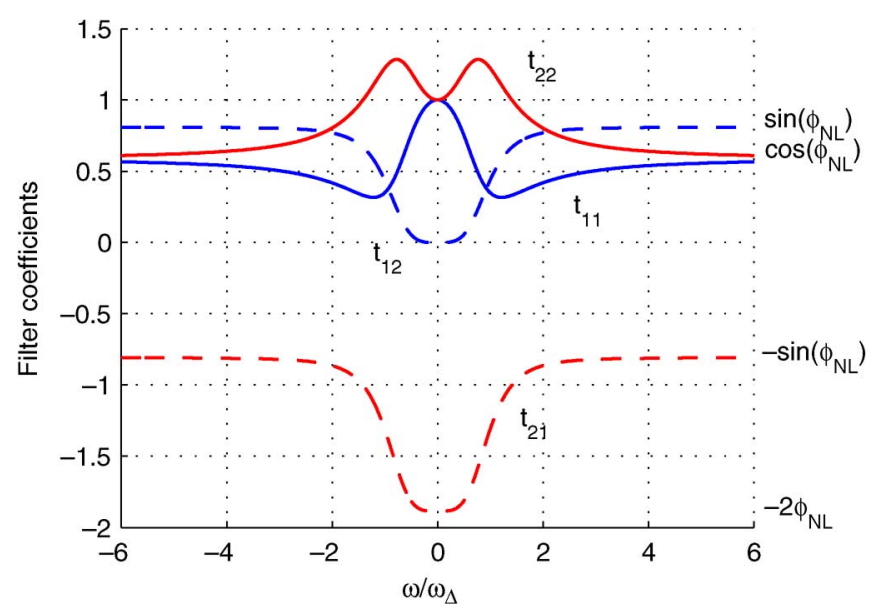

Fig. 8. Coefficients of the matrix T versus $\omega / \omega_{\Delta}$. System with $\Phi_{\mathrm{NL}}=0.3 \pi$ and $\xi_{\text {pre }}=\xi_{\text {in }}=\xi_{\text {post }}=0$.

get the photodetected current. Such a current is then low-pass filtered by the electrical filter before sampling.

Equation (29) can be generalized by including pre- and postcompensation. As (29) is a linear relation, the transfer matrix of the cascade of the prefiber, the optical link, and the postfiber is $\mathbf{T}_{\text {post }} \cdot \mathbf{T} \cdot \mathbf{T}_{\text {pre }}$, with $\mathbf{T}_{\text {pre }}$ and $\mathbf{T}_{\text {post }}$ being the transfer matrices defined as in (25) but evaluated for the pre- and postfiber, respectively. ${ }^{3}$ Since the propagation inside these fibers is assumed purely linear, $\mathbf{T}_{\text {pre }}$ and $\mathbf{T}_{\text {post }}$ simplify to the following orthogonal matrices:

$$
\mathbf{T}_{k}=\left[\begin{array}{cc}
\cos \left(\xi_{k} \omega^{2} / 2\right) & -\sin \left(\xi_{k} \omega^{2} / 2\right) \\
\sin \left(\xi_{k} \omega^{2} / 2\right) & \cos \left(\xi_{k} \omega^{2} / 2\right)
\end{array}\right]
$$

with $k \in\{$ 'pre', 'post' $\}$. Operator $\mathbf{T}_{k}$ rotates vector $\mathbf{v}$ by an angle $\hat{\theta}_{k}=\xi_{k} \omega^{2} / 2$.

In Fig. 8, we show the behavior of the four elements of matrix $\mathbf{T}$ versus $\omega / \omega_{\Delta}$, with $f_{\Delta}=\omega_{\Delta} / 2 \pi=1 / 2 \pi \sqrt{|S|}$ being the nonlinear diffusion bandwidth (with minor differences from the definition in [36]), i.e., the 3-dB bandwidth of the kernel $\widetilde{R}\left(\omega^{2}\right)$. The system had $\xi_{\text {pre }}=\xi_{\text {in }}=\xi_{\text {post }}=0$. We observe for $\omega \rightarrow \pm \infty$ a floor in each element, whose value is found by observing that the link matrix $\mathbf{T}$ approaches the orthogonal matrix having $\left[\cos \left(\Phi_{\mathrm{NL}}\right), \sin \left(\Phi_{\mathrm{NL}}\right)\right]$ as a first row. Except for the constant floor, we observe typical low-pass/bandpass behavior of all matrix elements over a single-sided bandwidth ${ }^{4}$ on the order of $f_{\Delta}$. The graphs in Fig. 8 change when including a nonzero cumulated GVD $\xi_{\text {tot }}=\xi_{\text {pre }}+\xi_{\text {in }}+\xi_{\text {post }} \neq 0$, since GVD scrambles the filter coefficients by adding frequency-chirped oscillations as indicated by (30).

\footnotetext{
${ }^{3}$ The choice of including the pre- and postfiber within the linearized model is due to the fact that, with correct pre- and postfiber choices, the distortions introduced by the DM link are reduced; hence the accuracy of the linearized model is expected to increase. The optical filter is instead kept out of the linear model, since its role is to filter out noise, thus increasing the distortion on the received field: its inclusion in the linear system matrix would worsen the accuracy of the linear model.

${ }^{4}$ The actual range over which the link matrix coefficients significantly differ from their floor (asymptotic) value depends also on the nonlinear phase, as given in [20], (34).
}

\section{A. Accuracy of Small-Signal Model}

We next show the accuracy of the small-signal DM-NLSE (29) by comparison with the SSFM solution of the NLSE.

We first analyzed a $20 \times 100 \mathrm{~km}$ DM terrestrial system with $R=40 \mathrm{~Gb} / \mathrm{s}, D_{\mathrm{T}}=8 \mathrm{ps} / \mathrm{nm} / \mathrm{km}$, (i.e., with $S=0.35$ ), and $\Phi_{\mathrm{NL}}=0.15 \pi$. The extinction ratio was $r=13 \mathrm{~dB}$. At the receiver, we used a Butterworth sixth-order OBPF with bandwidth $B_{o}=2 R$ and a Bessel fifth-order LPF with bandwidth $B_{e}=0.65 R$. Fig. 9 portrays the contour levels of the OSNR penalty at BER $=10^{-5}$, evaluated with the KL method [29], versus $\xi_{\text {pre }}$ and $\xi_{\text {in }}$, with $\xi_{\text {post }}$ optimized for each point by a numerical search, using the SSFM NLSE solution (top) and the small-signal DM-NLSE (bottom). The contours are spaced by $0.25 \mathrm{~dB}$, the innermost contour corresponding to $0 \mathrm{~dB}$ of penalty. The small-signal DM-NLSE qualitatively reproduces the SSFM NLSE contours, with a good quantitative match in the "optimal" region around the SLR (19) (also shown as a straight line) and a large overestimation of OSNR penalty away from such a region. Similar contours were obtained using a model based on pulse-broadening [25]. The important message here is that the contours based on the small-signal DM-NLSE are much faster to obtain than the SSFM-based contours, since a single fast-Fourier transform (FFT) is used for field propagation instead of the $\Phi_{\mathrm{NL}} / \Phi_{\mathrm{step}}$ FFTs required by the SSFM, with $\Phi_{\text {step }}$ being the nonlinear phase per step. In this case, $\Phi_{\text {step }}=$ $2 \times 10^{-3}$; hence the savings factor $\left(0.15 \pi / 2 \times 10^{-3} \simeq 235\right)$ is significant. The small-signal DM-NLSE can thus be effectively used as a fast design tool to quickly perform comparisons among DM designs and get a rough estimate of system performance.

We next analyzed a fully compensated $20 \times 100 \mathrm{~km}$ NRZ-OOK system, with $D_{\mathrm{T}}=8 \mathrm{ps} / \mathrm{nm} / \mathrm{km}, \xi_{\text {pre }}$ chosen according to the SLR (19), and $\xi_{\text {tot }}=0$. Receiver data were the same as before. In such a system, for increasing map strength $S$ (i.e., increasing bit rate), we measured the average $\Phi_{\mathrm{NL}}$ (7) that gives a reference eye closure penalty (ECP), i.e., the nonlinear threshold. We provide the NLT at both 1 and $3 \mathrm{~dB}$ of ECP, for both an unrealistically small extinction ratio of $r=1 \mathrm{~dB}$ (at which the small-signal model is plausible) and at a typical value $r=13 \mathrm{~dB}$.

In order to run accurate simulations, for each strength $S$ we used an appropriate number of bits $N_{\text {bit }}$ in the SSFM time-window, as will be discussed in Section VI-B, while the number of discrete points per bit was fixed to 32. Fig. 10 shows the measured threshold $\Phi_{\mathrm{NL}}$ normalized to $0.1 \pi$ and expressed in a decibel scale, $10 \log \left(\Phi_{\mathrm{NL}} /(0.1 \pi)\right)$. Since $\Phi_{\mathrm{NL}}$ is proportional to the launched power, penalties on $\Phi_{\mathrm{NL}}$ in a decibel scale correspond to power penalties on the same scale. The figures show that the NLT is a decreasing function of $S$ due to the increasing GVD-SPM interaction along the system, which, in the DM-NLSE jargon, corresponds to a stronger filtering effect introduced by the kernel. At $r=1 \mathrm{~dB}$, the SSFM and (29) almost coincide, as expected. At $r=13 \mathrm{~dB}$, the small-signal DM-NLSE NLT only slightly underestimates (less than $0.5 \mathrm{~dB}$ ) the true (SSFM) NLT at small strengths up to about $S \cong 0.3$ (corresponding here to $R \cong 40 \mathrm{~Gb} / \mathrm{s}$, and consistently with the observed slight overestimation of distortions in Fig. 9). Over such a range, Fig. 10 (bottom) shows that the underestimation 

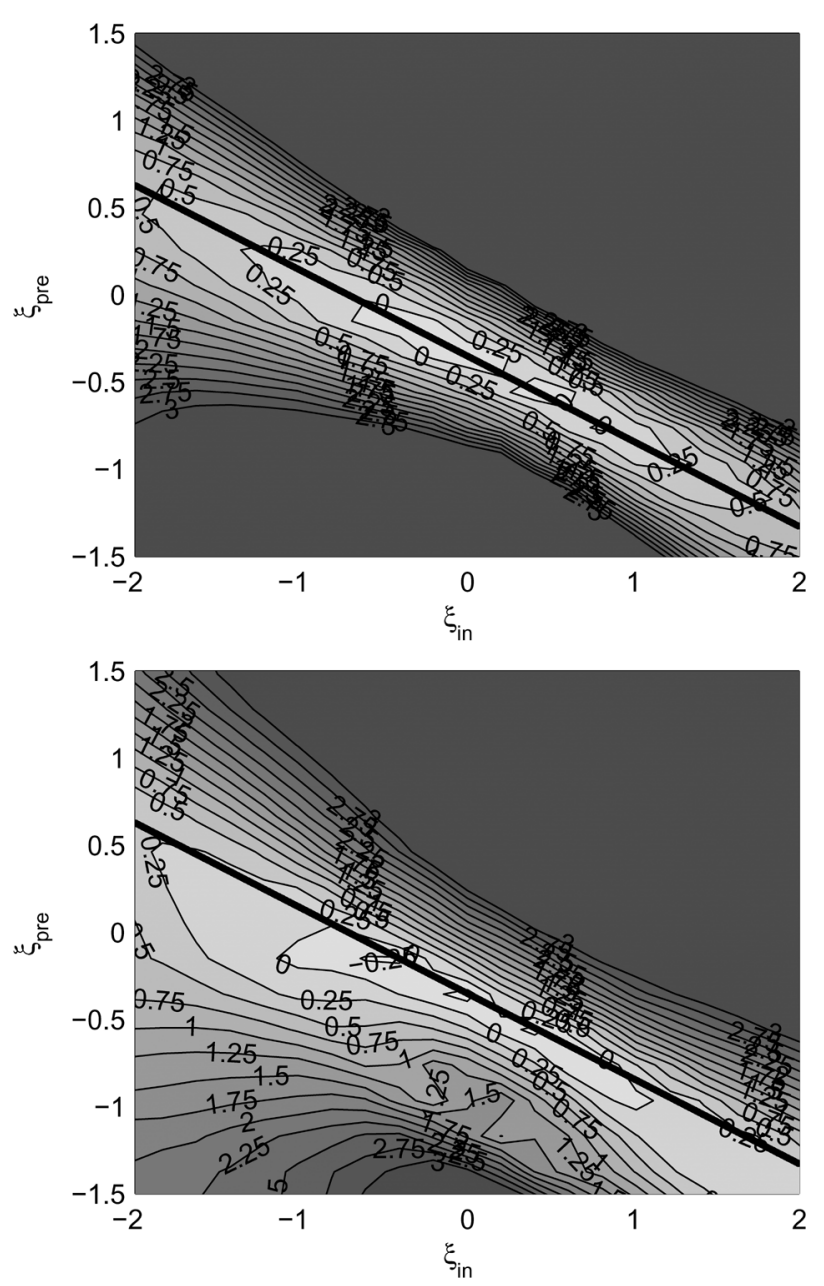

Fig. 9. OSNR penalty atBER $=10^{-5}$ versus $\xi_{\text {pre }}$ and $\xi_{\text {in }}$, optimized $\xi_{\text {post }}$. NRZ-OOK link with $20 \times 100 \mathrm{~km}, D_{\mathrm{T}}=8 \mathrm{ps} / \mathrm{nm} / \mathrm{km}, R=40 \mathrm{~Gb} / \mathrm{s}$ (i.e., $S=0.35$ ), and $\Phi_{\mathrm{NL}}=0.15 \pi$. (Top) SSFM simulation. (Bottom) Small-signal model. (Solid straight lines) SLR (19).

increases (but remains less than $1 \mathrm{~dB}$ ) if we move to larger distortions $(\mathrm{ECP}=3 \mathrm{~dB})$. Beyond $S \cong 0.3$, i.e., at stronger DM, we note that the small-signal DM-NLSE overestimates instead the NLT by an increasing amount, in excess of $3 \mathrm{~dB}$ at $S \cong 2$, and the small-signal NLT at $r=13 \mathrm{~dB}$ tends to coincide with that at $r=1 \mathrm{~dB}$.

The under- and overestimation of the small-signal DM-NLSE with respect to the true SSFM NLSE solution is the net effect of two effects: the large-signal DM-NLSE approximation of the true NLSE and the departure of the small-signal response from the large signal response. To understand the causes of discrepancy, one can take a look at the time waveforms at specific operating points. For instance, Fig. 11 shows the normalized received power after the optical filter that yields ECP $=$ $1 \mathrm{~dB}$ for $S=0.022$ and $S=0.35$ (i.e., $R=10 \mathrm{~Gb} / \mathrm{s}$ and $R=40 \mathrm{~Gb} / \mathrm{s}$ at $D_{\mathrm{T}}=8 \mathrm{ps} / \mathrm{nm} / \mathrm{km}$, respectively), for both the true (SSFM) NLSE solution (symbols) and the DM-NLSE small-signal model (solid) at $r=13 \mathrm{~dB}$. Such a figure confirms the good match observed in Fig. 10 in the small strength case. At $R=40 \mathrm{~Gb} / \mathrm{s}$, there are some discrepancies, in particular a severe overestimation on the spaces by the small-signal
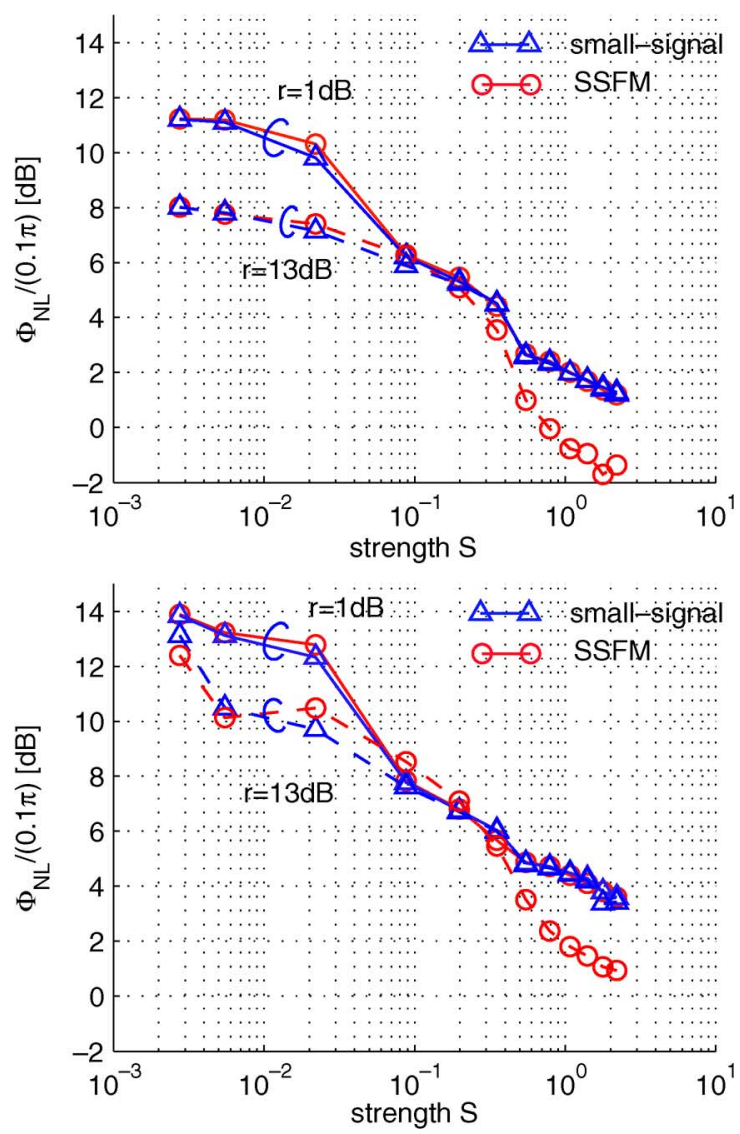

Fig. 10. $\Phi_{\mathrm{NL}}$ that gives (top) $\mathrm{ECP}=1 \mathrm{~dB}$ and (bottom) $\mathrm{ECP}=3 \mathrm{~dB}$ versus map strength $S$ in a fully compensated system, SLR-optimized $\xi_{\text {pre }}$, and $\xi_{\text {tot }}=$ 0. (Circles) SSFM NLSE solution; (triangles) small-signal DM-NLSE.

DM-NLSE, which justifies the observed more conservative estimation of the NLT. This failure can be associated with the growth of ghost pulses, which are incorrectly reproduced by the small-signal model. The reason can be understood by observing the step-response of the DM-link in the time domain. Since the step signal has odd symmetry in the time domain with respect to its average value, and the DM-link matrix has real coefficients even in $\omega$, and hence time-even impulse responses, the response of the linearized system will preserve the odd symmetry of the input step. On the contrary, the true step-response of the DM-NLSE is strongly asymmetric, as already noted in Section IV-A and as well captured by the large-signal perturbative analysis, which is thus more accurate than the small-signal analysis.

\section{ImPliCATIONS OF THE SMALL-SignAl MODEL}

In this section, we use the small-signal DM-NLSE model to provide an alternative derivation of the SLR (19) already proven with a large-signal perturbative analysis of the DM-NLSE and to show a practical expression of the minimum PRBS length scaling rule in optimized DM terrestrial links.

\section{A. Optimal Precompensation}

A singular value decomposition (SVD) [37] of system matrix (25) gives $\mathbf{T}=\mathbf{U} \Sigma \mathbf{V}^{T}$, where $\mathbf{U}$ and $\mathbf{V}$ are the orthogonal matrices that diagonalize $\mathbf{P}=\mathbf{T} \mathbf{T}^{T}$ and $\mathbf{Q}=\mathbf{T}^{T} \mathbf{T}$, respectively, 

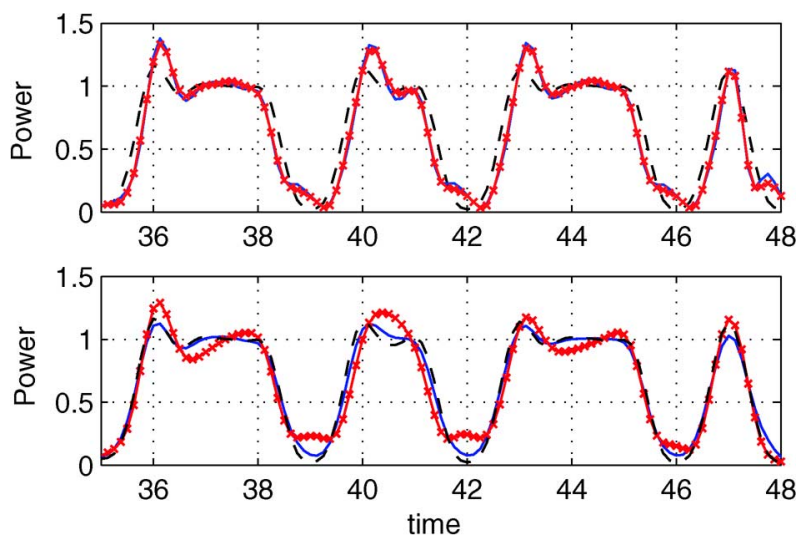

Fig. 11. Received power versus normalized time at (top) $S=0.022$ and (bottom) $S=0.35$ at ECP $=1 \mathrm{~dB} . \xi_{\text {in }}=0$, SLR-optimized $\xi_{\text {pre }}, \xi_{\text {tot }}=0$. (Dashed lines) Back-to-back; (symbols) SSFM NLSE solution; (solid lines) small-signal DM-NLSE.

and $\boldsymbol{\Sigma}$ is a diagonal matrix whose nonzero elements are the nonnegative square roots of the eigenvalues of $\mathbf{P}$. Being orthogonal, matrices $\mathbf{U}$ and $\mathbf{V}$ can be expressed as in (30) and uniquely identified by their rotation angle $\hat{\theta}_{u}$ and $(\pi / 2)+\hat{\theta}_{v}$, respectively, where $\hat{\theta}_{u}(\omega)$ is related to the entries $\left\{P_{i j}\right\},(i, j) \in(1,2)$ of matrix $\mathbf{P}$ as

$$
\hat{\theta}_{u}(\omega)=\frac{1}{2} \arctan \left(\frac{2 P_{12}}{P_{11}-P_{22}}\right)
$$

and similarly for the rotation angle $\hat{\theta}_{v}(\omega)$ using the entries of $\mathbf{Q}$ instead of $\mathbf{P}$. Both $\hat{\theta}_{u, v}$ are positive for counterclockwise rotations. The eigenvalues of $\boldsymbol{\Sigma}=\operatorname{diag}\left(\left[\sigma_{m}, \sigma_{M}\right]\right)$ are

$$
\sigma_{M, m}=\sqrt{\frac{P_{11}+P_{22}}{2} \pm \frac{1}{2} \sqrt{\left(P_{11}-P_{22}\right)^{2}+4 P_{12}^{2}}} .
$$

If we include in the analysis the orthogonal pre- and postfiber matrices (30), the overall system transfer matrix is $\mathbf{T}_{\text {post }} \mathbf{U} \boldsymbol{\Sigma} \mathbf{V}^{T} \mathbf{T}_{\text {pre. }}$ Closer examination of this global SVD reveals that, from a geometric point of view, the particular vector $\mathbf{v}(0, \omega)=[1,0]^{T}$ at frequency $\omega$ undergoes i) a rotation by an angle $\xi_{\text {pre }} \omega^{2} / 2-\pi / 2-\hat{\theta}_{v}(\omega)$, then ii) a stretching due to $\sigma_{m, M}$, and iii) a final rotation by an angle $\hat{\theta}_{u}(\omega)+\xi_{\text {post }} \omega^{2} / 2$. After such steps, the input vector has experienced only SPM if the eigenvalues are constant in $\omega$ and the rotation angles $\hat{\theta}_{u, v}(\omega)$ are canceled by the pre- and postfibers. It is impossible to meet such conditions exactly: hence we try to approximate them through a polynomial expansion of (31) and (32). Since the pre- and postrotation angles are quadratic in $\omega$, we stop such an expansion up to $\omega^{2}$. After evaluating $\mathbf{P}$ and $\mathbf{Q}$ from (25), we obtain the following approximation for the angles $\hat{\theta}_{u}(\omega)$ and $\hat{\theta}_{v}(\omega)$

$\hat{\theta}_{u, v}(\omega) \cong \pm \frac{1}{2} \operatorname{arccot}\left(\Phi_{\mathrm{NL}}\right)+\frac{\omega^{2}}{2}\left[-S \pm \frac{\xi_{\mathrm{in}}}{6}\left(\frac{3+2 \Phi_{\mathrm{NL}}^{2}}{1+\Phi_{\mathrm{NL}}^{2}}\right)\right]$

where the top signs apply to $\hat{\theta}_{u}$ and the bottom ones to $\hat{\theta}_{v}$. The parts of $\hat{\theta}_{u}$ and $\hat{\theta}_{v}$ quadratic in $\omega$ can be nulled by the following optimal $\xi_{\text {pre }}$ and $\xi_{\text {post }}$ values:

$$
\xi_{\text {pre,post }}=\mp S-\frac{\xi_{\text {in }}}{6}\left(\frac{3+2 \Phi_{\mathrm{NL}}^{2}}{1+\Phi_{\mathrm{NL}}^{2}}\right)
$$

where the top - sign is for the optimal $\xi_{\text {pre }}$. Such conditions equalize the rotation matrices $\mathbf{U}$ and $\mathbf{V}$ up to second order in $\omega$, but an $\omega$-dependence of the eigenvalues still remains. After a Taylor expansion in $\omega, \sigma_{M, m}$ turn out to be

$$
\sigma_{M, m} \cong\left(\sqrt{1+\Phi_{\mathrm{NL}}^{2}} \pm \Phi_{\mathrm{NL}}\right)\left(1 \pm \frac{\Phi_{\mathrm{NL}}^{2}}{\sqrt{1+\Phi_{\mathrm{NL}}^{2}}} \frac{\xi_{\mathrm{in}}}{6} \omega^{2}\right)
$$

However, in the limit of $\xi_{\text {in }} \Phi_{\mathrm{NL}}^{2} \rightarrow 0$, the eigenvalues (35) and the net rotation angles of the optimized global SVD do approach those of a system with only SPM. In such a limit, (34) coincides with the SLR (19) derived in the large-signal regime. Such an equivalence is another indicator, besides the contour evaluations at Fig. 9, that DM optimizations performed on the small-signal DM-NLSE lead to very similar choices as those performed with a large-signal analysis but with a much faster numerical evaluation.

\section{B. Memory of the Optical Link}

Closer examination of Fig. 8, obtained at $\xi_{\text {in }}=\xi_{\text {pre }}=$ $\xi_{\text {post }}=0$, reveals that the entries of matrix $\mathbf{T}$ in (25) have the form of a constant added to functions of finite bandwidth. Hence their inverse Fourier transform can reasonably be described by functions of finite temporal duration, which can be taken as the "memory" of the optical link. Fig. 12 clarifies this statement by showing the response of an $R=100 \mathrm{~Gb} / \mathrm{s}$ DM-link to the sequence ...1110111..., which well approximates the response to a negative Dirac's delta. We used a link with transmission fiber dispersion $D_{\mathrm{T}}=17 \mathrm{ps} / \mathrm{nm} / \mathrm{km}(S=4.67)$, fully compensated at each span without pre- and postfibers, at an average nonlinear phase $\Phi_{N L}=0.2 \pi$. The dashed line in the figure is the transmitted power, the solid line the power predicted by the small-signal model (29), and the circles SSFM simulations. First observe that, since the single space buried in a long string of marks produces a very small perturbation of the power feeding the link, the small-signal model is expected to give very reliable results. From (25), at $\xi_{\text {in }}=0$, the IM-IM filter is $t_{11}(\omega)=\cos \eta-(\sin \eta / \eta)\left(\Phi_{N L} S \omega^{2} / 1+\right.$ $\left.S^{2} \omega^{4}\right)$, with $\eta=\Phi_{\mathrm{NL}} S \omega^{2} / \sqrt{1+S^{2} \omega^{4}}$. At small $\Phi_{N L}$, i.e., small $\eta, t_{11}(\omega) \cong 1-\Phi_{\mathrm{NL}} \operatorname{sgn}[S]\left(|S| \omega^{2} / 1+\left(S \omega^{2}\right)^{2}\right)$, whose inverse Fourier transform is

$$
t_{11}(\tau)=\delta(\tau) \pm \frac{\Phi_{\mathrm{NL}} e^{-\frac{|\tau|}{\sqrt{2|S|}}}}{2 \sqrt{2|S|}}\left(\cos \frac{|\tau|}{\sqrt{2|S|}}-\sin \frac{|\tau|}{\sqrt{2|S|}}\right)_{36)}
$$

with the sign + for $S<0$ and the sign - for $S>0$. We verified (36) to match well with the shown small-signal response to the delta spike. The impulse response dies away in slightly more than \pm 3 time constants, i.e., over a time window of $T_{m}=$ $6 \sqrt{2|S|} \sim 10 \sqrt{S}$, as we verify from the figure, and consistently with twice the value of the duration of the perturbation in the large-signal step response reported in Fig. 6.

Knowledge of $T_{m}$ is mandatory to correctly reproduce all the dominant intrachannel distortions in an experimental or numerical setup. If $2^{n}-1$ is the length of the transmitted PRBS, then $n$ must exceed $m=\left\lceil T_{m} d\right]$ bit slots in order to reproduce all significant intersymbol interference (ISI) patterns, with $d$ being 


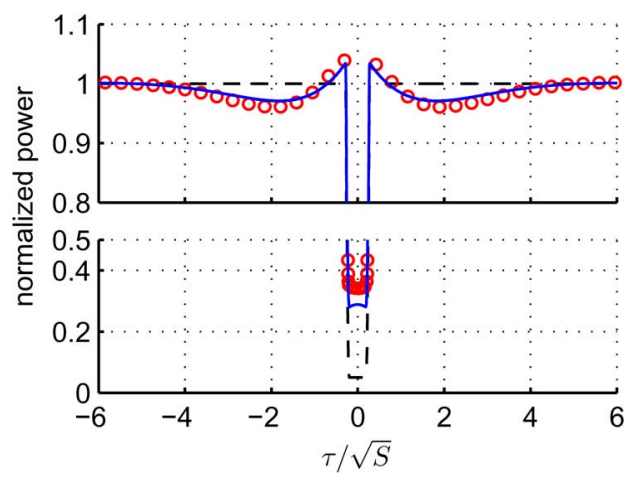

Fig. 12. Response to the $\ldots 1110111 \ldots$ sequence versus $\tau / \sqrt{S}$. (Circles) SSFM simulations with $\Phi_{N L}=0.2 \pi, \xi_{\text {in }}=\xi_{\text {pre }}=\xi_{\text {post }}=0, S=4.67$; (solid line) model (29); d(ashed line) transmitted power.

the pulse duty cycle and $\lceil x\rceil$ the smallest integer larger than $x$. We call $m$ the ISI depth.

We now look for an expression of $T_{m}$ in the general case of nonzero pre-, post-, and inline compensation. Following the reasoning in Section VI-A, $T_{m}$ will depend on the memory introduced by the orthogonal matrices $\mathbf{P}$ and $\mathbf{Q}$ and that introduced by the eigenvalues of $\boldsymbol{\Sigma}$. With an optimal choice of the pre- and postfibers as shown in Section VI-A, the rotation matrices $\mathbf{T}_{\text {post }} \mathbf{U}$ and $\mathbf{V}^{T} \mathbf{T}_{\text {pre }}$ are frequency independent to $\omega^{2}$ order , and the main contribution to the system memory comes from the system eigenvalues (32). Since there are two eigenvalues, we define the system memory as their largest time-duration. As such a duration is not exactly finite, several definitions can be introduced, e.g., the full-width at half-maximum duration, the inverse of the $3-\mathrm{dB}$ bandwidth, the $1 / e$ duration, etc. Usually, the difference among these criteria reduces to a constant multiplicative factor, which can be found a posteriori with a calibration of the method by comparison with numerical results. After such a calibration, the most important information that can be drawn from the memory time derives from its functional behavior and its scaling properties with system parameters. For the sake of simplicity, we define here the memory time to be $T_{m}=2 / B_{3}$, where $B_{3}$ is the smallest $3-\mathrm{dB}$ bandwidth of the eigenvalues. Such a bandwidth is implicitly defined as $\sigma_{s b}^{2}\left(\omega_{3}\right)=(1 / 2)\left(\sigma_{s b}^{2}(0)+\sigma_{s b}^{2}(\infty)\right)$, where $\omega_{3}=2 \pi B_{3}$ and $\sigma_{s b}$ is the eigenvalue with the smallest bandwidth. This equation can be quickly solved numerically. An analytic approximation of $B_{3}$ can also be found by means of a first-order Taylor expansion in $\Phi_{\mathrm{NL}}$ of the eigenvalues (32)

$$
\sigma_{M, m} \cong 1 \pm \sqrt{2} \sqrt{\frac{1-\cos \left(\omega^{2} \xi_{\text {in }}\right)}{\left(1+S^{2} \omega^{4}\right) \omega^{4} \xi_{\text {in }}^{2}}} \Phi_{\mathrm{NL}} .
$$

For moderate values of the inline dispersion $\omega_{3}^{2} \xi_{\text {in }} \ll 1$, we approximate $\cos \left(\omega_{3}^{2} \xi_{\text {in }}\right) \cong 1-\omega_{3}^{4} \xi_{\text {in }}^{2} / 2$ in (37), yielding, for $B_{3}$

$$
B_{3}=\frac{\sqrt{3}}{2 \pi\left(3 S^{2}+\xi_{\text {in }}^{2}\right)^{1 / 4}}
$$

from which we derive the ISI depth as

$$
m=\left\lceil\frac{4 \pi d}{\sqrt{3}}\left(3 S^{2}+\xi_{\text {in }}^{2}\right)^{1 / 4}\right\rceil .
$$

Such a formula holds when $\omega_{3}^{2} \xi_{\text {in }} \ll 1$, i.e., when $\left|\xi_{\text {in }}\right| \ll 0.6|S|$. Since both $S$ and $\xi_{\text {in }}$ are proportional to $R^{2}$ (see Appendix I), a main implication of (38) is that $m$ scales as $O(R)$. Hence, the memory of the nonlinear effects scales more slowly than the memory of pure GVD, which is $O\left(R^{2}\right)$, as we simply prove in Appendix II. In the context of the small-signal model, the eigenvalues of $\Sigma$ with only GVD are equal to one; thus the memory time arises only from the orthogonal matrices $\mathbf{P}$ and $\mathbf{Q}$, whose entries are GVD chirped sinusoids, as can be deduced by their expression in (30).

In conclusion, for an optimized DM link with moderate in-line dispersion $m$ scales as $O(R)$, while for DM systems dominated by GVD, one should also take into account the additional memory due to GVD, which scales as $O\left(R^{2}\right)$.

To test (38) for optimized DM terrestrial systems, we investigated through SSFM simulations the performance of a single NRZ-OOK channel propagating into a $D_{\mathrm{T}}=8 \mathrm{ps} / \mathrm{nm} / \mathrm{km}$, $20 \times 100 \mathrm{~km}$ terrestrial DM link by varying either the strength $S$ or the inline dispersion $\xi_{\text {in }}$, and always optimizing $\xi_{\text {pre }}$ and $\xi_{\text {post }}$. We get information on memory by measuring the nonlinear threshold $\Phi_{\mathrm{NL}}$ that gives $1 \mathrm{~dB}$ of OSNR penalty versus back-to-back at a bit error rate $\mathrm{BER}=10^{-5}$ for increasing PRBS lengths. In this way, the nonlinear distortion remains comparable for all cases. The BER was evaluated with the KL algorithm. First we checked the impact of strength $S$ by analyzing a fully compensated system. We used variable strengths in the range $S=0.022 \div 8.8$, corresponding to $R=10 \div 200 \mathrm{~Gb} / \mathrm{s}$. The nonlinear threshold was searched in the range $\Phi_{\mathrm{NL}}=(0.1 \pi \div 0.4 \pi)$ at $S=0.35$, and that range was scaled by $1 / \sqrt{S}$ when varying the strength in agreement with the growth of ghost pulses found in Section IV-A. The precompensation followed the SLR (19), while the postcompensation was optimized for each $S$ using a PRBS of $2^{17}$ bits, which was our computational limit. Fig. 13 gives the nonlinear threshold versus $S$ and versus the logarithmic PRBS length $n$. The threshold is given in a decibel scale as $10 \log _{10}\left(\Phi_{N L} /(0.1 \pi)\right)$. The solid line with circles plots (38) and captures quite reasonably the edge of the stable zone of the contour, i.e., the one with horizontal levels, where the threshold is independent of the PRBS length.

In Fig. 14, we fixed $\Phi_{N L}=0.15 \pi$ and $R=40 \mathrm{~Gb} / \mathrm{s}$ and measured the OSNR penalty by varying the cumulated inline dispersion. The top graphs refer to $D_{\text {in }}=$ $-70,-60,-40,-20,0 \mathrm{ps} / \mathrm{nm} / \mathrm{span}$ (i.e., $\xi_{\text {in }}=-2.85 \div 0$ ), the bottom graphs to $D_{\text {in }}=0,20,40,60,70 \mathrm{ps} / \mathrm{nm} / \mathrm{span}$. Here we set $\xi_{\text {tot }}=0.1$, which is close to the optimal value for each $\xi_{\text {in. }}$. The solid lines refer to SSFM simulations, while the dashed lines with circles refer to (38). We note that (38) gives a reasonable estimation of DM system memory whenever the inline dispersion does not exceed $\pm 40 \mathrm{ps} / \mathrm{nm}$, i.e., when $\left|\xi_{\text {in }}\right| \lesssim 5|S|$, which shows that (38) holds over a larger range than that expected from its derivation.

The penalty curves in Fig. 15, obtained with SSFM simulation, clarify the need for bit-sequences of sufficient length for penalty measurements. The monotonically increasing solid line with squares shows the ECP versus map strength $S$ for the same system as in Fig. 13. For convenience, we provide a translation of the $S$ axis into a bit-rate axis for a system with 


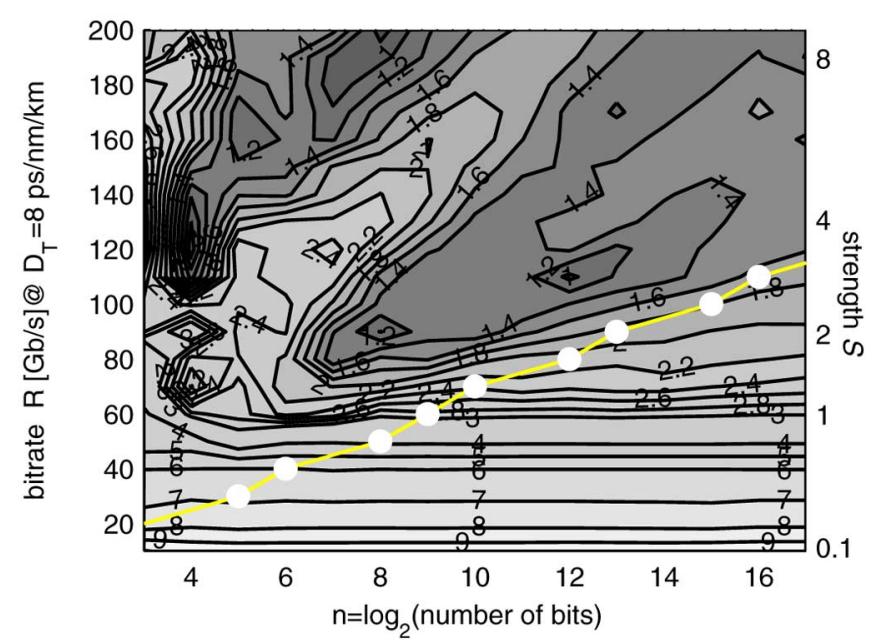

Fig. 13. Threshold (dB) that gives $1 \mathrm{~dB}$ of OSNR penalty at BER $=10^{-5}$ versus $S$ and $n$ with full in-line compensation. (Circles) (38).
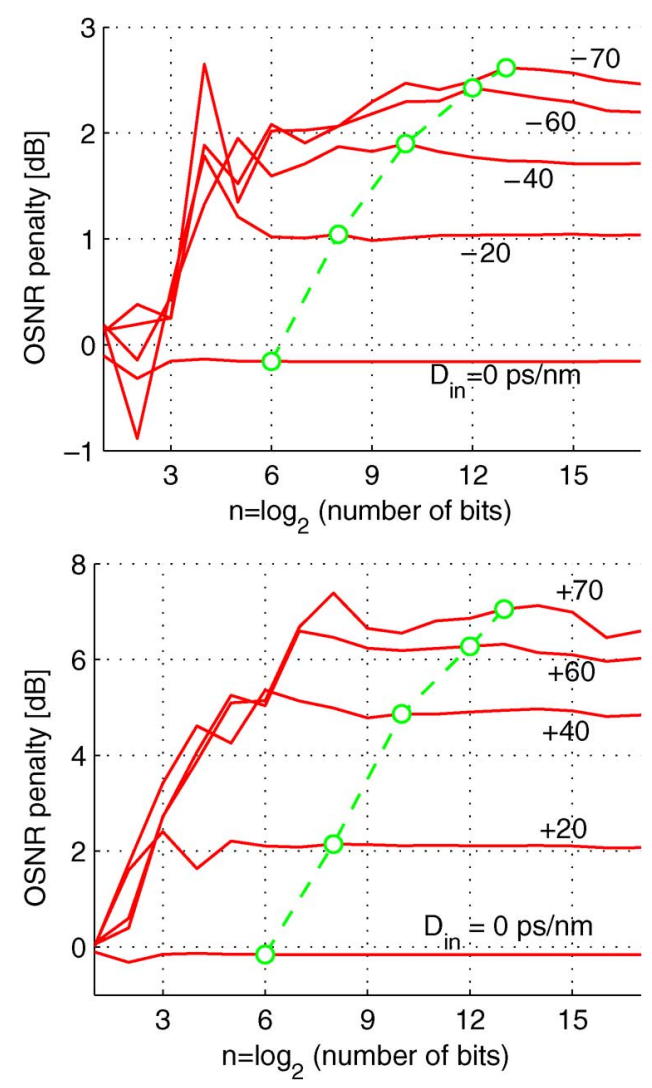

Fig. 14. OSNR penalty versus $n$ for various inline dispersions. (Circles) (38). $R=40 \mathrm{~Gb} / \mathrm{s}$.

$D_{\mathrm{T}}=8 \mathrm{ps} / \mathrm{nm} / \mathrm{km}$. We used a transmitted peak power equal to $1.6 \mathrm{~mW}$ (peak $\Phi_{\mathrm{NL}}=0.4 \pi$ ). The penalty was evaluated using, for each value of $S$, a PRBS of length as in (38). With a solid line, we show the ECP evaluated at the same peak $\Phi_{\mathrm{NL}}$ using a fixed PRBS of 128 bits for all $S$, which produces the artifact of an apparent ECP resonance at a strength value close to $S=1$ [8], [10], [11]. Such an artifact is clearly due to the short sequence length, which does not reproduce the main distorting patterns. For instance, patterns that cause the ECP to monotonically increase with $S$ are the step sequence ...000111...

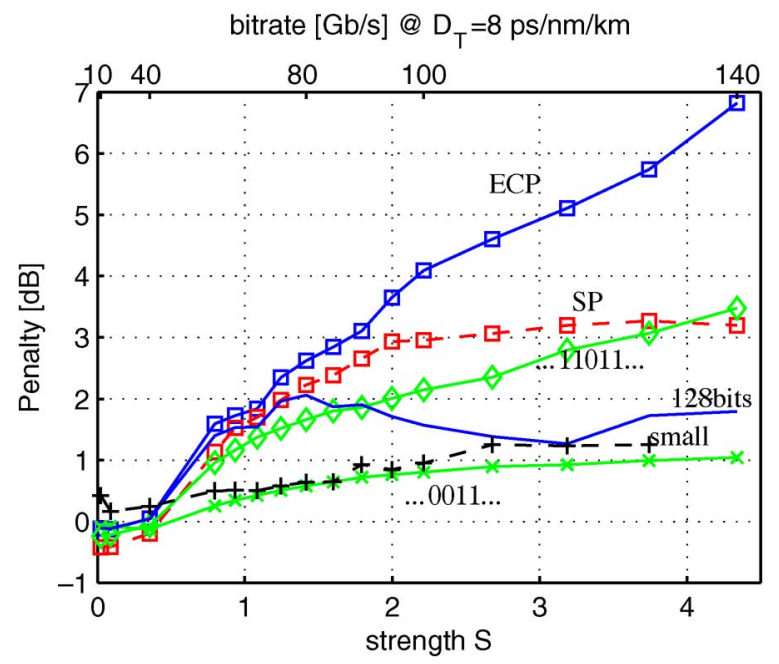

Fig. 15. Penalties versus $S$ for same system as Fig. 13. ECP/SP: eye-closure penalty/OSNR penalty when using $2^{m}$ bits, $m$ as in (38); 128 bits: ECP when using a fixed PRBS of $2^{7}$ bits; . . 0011 . . and . . 11011 . . ECP when using step and delta sequences propagated in a SSFM window of 256 bits. "Small": ECP using (29). Peak $\Phi_{\mathrm{NL}}=0.4 \pi$. $\xi_{\text {tot }}$ optimized for the SP curve.

and the "negative-delta" sequence ...1110111..., whose ECP evaluated at the same peak $\Phi_{\mathrm{NL}}=0.4 \pi$ and with 256 bits in the SSFM window [a number much larger than that needed as per (38) over the shown range of $S$ ] is shown in the same figure. Note that the ECP of the step sequence grows as $\log (S)$, i.e., the penalty in a linear scale grows linearly with $S$, as expected from the linear growth of ghost pulse energy derived in Section IV-A, while the ECP of the negative-delta sequence is almost twice that of the step sequence. With a dashed line with crosses we show the ECP obtained with the small-signal DM model (29) using PRBS of appropriate length as in (38). Once again, the model does not predict the ECP resonance artifact.

Finally, from a comparison of the OSNR penalty at BER = $10^{-5}$ (curve labeled SP) and the corresponding ECP, both using a PRBS of length (38), we note that, at large strength, the OSNR penalty grows more slowly than the ECP because the impact on BER of the bad sequences that set the ECP is statistically smaller.

\section{CONCLUSIONS}

The aim of this paper was to show that the DM-NLSE provides all the necessary information needed to optimize not only very-long-haul periodic submarine systems but also terrestrial DM systems with a limited number of spans. All major engineering optimization rules and scaling laws for nonlinear effects empirically found in the past for terrestrial systems, along with novel results on system memory, can be straightforwardly derived from the DM-NLSE, especially from its kernel. We have shown that nonlinear phase and map strength are the only two parameters determining the terrestrial link kernel. We have seen a quick derivation of the straight-line rule for the optimal precompensation based on a perturbative solution of the DM-NLSE. In the process, we have realized that with the optimal precompensation, the DM system behaves as a system with only SPM, so that subsequent optimization of the postcompensation follows a well-known rule for pulse compression [33]. We have 
developed a linear model for a quick qualitative analysis of DM systems based on the OOK format. A remark about the small-signal DM-NLSE model regards the choice of the power $P$ in (28). Such a value was chosen equal to the average power evaluated over the entire time axis. However, the distortions affect the signal only within a time window $T_{m}$. Hence, for each sampling time, a specific power $\bar{P}$ averaged over a window $T_{m}$ exists, a fact that leads to a time-varying model. We applied such an idea in [31] to evaluate the ASE power spectral density for RZ-DPSK format at the sampling epochs. However, since fundamental results like (19) and (38) showed independence from $\Phi_{\mathrm{NL}}$, we conclude that the time-invariant model is sufficient to derive optimal design rules.

Perhaps most importantly, in this paper, we have quantified the intersymbol interference depth of an optimized DM terrestrial link, making it clear that whenever dispersion is compensated at each span down to moderate amounts, the ISI depth is not as large as in systems in which dispersion is compensated only at the link end, as in highly dispersed transmission.

The DM-NLSE can be extended to multichannel transmissions, and some results have already been reported [26], while the many details of the theory of WDM DM-NLSE will be published elsewhere. WDM DM-NLSE is the ultimate tool that allows one to bring together in a systematic framework all known results about nonlinear propagation in DM optical links.

\section{APPENDIX I}

\section{FROM STANDARD TO NORMALIZED UNITS}

Throughout this paper, we use dimensionless units such as the map strength $S$ and the inline dispersion $\xi_{\text {in }}$. To relate them to the standard dimensional units, we use a reference time $T$ equal to the pulse duration $T=d / R$, with $d$ being the pulse dutycycle and $R$ the bit rate. For the considered case of ultralong terrestrial system diagrammed in Fig. 1, we have the following identities:

$$
\begin{aligned}
S & =-\frac{L_{A}}{L_{\Delta}}=\frac{\lambda^{2}}{2 \pi c}\left(\frac{R}{d}\right)^{2} \frac{1}{\alpha}\left(D_{\mathrm{T}}-\frac{D_{\mathrm{in}}}{L}\right) \\
\xi_{\text {in }} & =\frac{\lambda^{2}}{2 \pi c}\left(\frac{R}{d}\right)^{2} N_{s} D_{\text {in }}
\end{aligned}
$$

where $D_{\mathrm{T}}[\mathrm{ps} / \mathrm{nm} / \mathrm{km}]$ and $\alpha\left[\mathrm{m}^{-1}\right]$ are the chromatic dispersion coefficient and the attenuation of the transmission fiber, respectively, $D_{\text {in }}[\mathrm{ps} / \mathrm{nm}]$ is the inline residual dispersion per span, $N_{s}$ the number of spans, $c$ the speed of light, and $\lambda$ the channel wavelength. Note that both $S$ and $\xi_{\text {in }}$ have opposite sign to their corresponding dispersion lengths $L_{\Delta}$ and $L_{D}$, respectively, which have the sign of $\beta_{2}$.

For a numerical feeling, an NRZ system with $D_{\mathrm{T}}=$ $8 \mathrm{ps} / \mathrm{nm} / \mathrm{km}$ at $R=10 \mathrm{~Gb} / \mathrm{s}$ corresponds to a strength $\mathrm{S}=0.022$. Since $S$ scales as $R^{2}$, at $40 \mathrm{~Gb} / \mathrm{s}$ the strength is 16 times larger, $S \cong 0.35$. A value of $\xi_{\text {in }}=0.1$ corresponds to $800 \mathrm{ps} / \mathrm{nm}$ at $10 \mathrm{~Gb} / \mathrm{s}$, while it decreases to $50 \mathrm{ps} / \mathrm{nm}$ at 40 $\mathrm{Gb} / \mathrm{s}$. The cumulated dispersions within the pre- and postcompensating fiber, i.e., $\xi_{\text {pre }}$ and $\xi_{\text {post }}$, follow the same conversion rule as for $\xi_{\text {in }}$, that is, $\xi_{\text {pre } / \text { post }}=\left(\lambda^{2} / 2 \pi c\right)(R / d)^{2} D_{\text {pre/post }}$, with $D_{\text {pre/post }}$ in $[\mathrm{ps} / \mathrm{nm}]$. Since in this paper we assumed long spans and purely linear compensating fibers, the nonlinear phase cumulated by a constant signal of power $P[\mathrm{~mW}]$ is from (7): $\left.\Phi_{\mathrm{NL}}=N_{s} P L<\gamma(z) f(z)\right\rangle=N_{s} P \gamma / \alpha$, where $\gamma\left[\mathrm{m}^{-1} \mathrm{~mW}^{-1}\right]$ is the nonlinear coefficient of the transmission fiber.

\section{APPENDIX II \\ THE MEMORY OF PURE GVD}

The impulse response of a purely linear fiber that accumulates a normalized dispersion $\xi$ is $h(\tau)=\exp \left(-j \tau^{2} /(2 \xi)\right) / \sqrt{2 \pi j \xi}$ and is even in (normalized) time $\tau$. Its real and imaginary components are chirped sinusoids, with instantaneous frequency $f_{\mathrm{n}}(\tau) \triangleq(1 / 2 \pi)|(d \arg [h(\tau)] / d \tau)|=|\tau| /(2 \pi|\xi|)$ increasing with time $|\tau|$. If the input field $A(\tau)$ is an OOK NRZ real signal with bit time $T=1$, the output field at the sampling time $\tau_{0}$ (usually the bit center) is the convolution $y\left(\tau_{0}\right)=(1 / \sqrt{2 \pi j \xi}) \int_{-\infty}^{\infty} \exp \left(-j\left(\tau_{0}-\tau\right)^{2} /(2 \xi)\right) A(\tau) d \tau$. The integrand is the product of the input field $A(\tau)$ and the chirped sinusoids centered at the sampling time $\tau_{0}$. All the NRZ bit pulses in $A(\tau)$ over which the chirped sinusoids have many cycles (in practice, more than three cycles) do not contribute significantly to the integral in the sampled output $y\left(\tau_{0}\right)$. Hence the significant bits extend for a duration $\pm \tau_{m}$ around the sampling time $\tau_{0}$, such that $f_{\mathrm{n}}\left(\tau_{m}\right) \geq 3$. This gives $\tau_{m}=3 \cdot 2 \pi|\xi|$, and thus the "memory" due to GVD can be approximated as the rounded number of bits $m \sim\left\lceil 2 \tau_{m}\right\rceil=\lceil 12 \pi|\xi|\rceil$, which proves that the ISI depth for pure GVD scales as $O\left(R^{2}\right)$, like the term $|\xi|$. Such a scaling is in agreement with the scaling of the broadening factor of a Gaussian pulse [22].

\section{ACKNOWLEDGMENT}

The authors would like to thank J.-C. Antona and S. Bigo of Alcatel-Lucent for motivation, stimulating discussions, and continued support.

\section{REFERENCES}

[1] M. J. Ablowitz and G. Biondini, "Multiscale pulse dynamics in communication systems with strong dispersion management," Opt. Lett., vol. 23, pp. 1668-1670, Nov. 1998.

[2] M. J. Ablowitz and T. Hirooka, "Managing nonlinearity in strongly dispersion-managed optical pulse transmission," J. Opt. Soc. Amer. B, Opt. Phys., vol. 19, no. 3, pp. 425-439, Mar. 2002.

[3] M. J. Ablowitz and T. Hirooka, "Resonant intrachannel pulse interactions in dispersion-managed transmission systems," IEEE J. Sel. Topics Quantum Electron., vol. 8, pp. 603-615, May/Jun. 2002.

[4] H. A. Haus, "Quantum noise in a soliton like repeater system," J. Opt. Soc. Amer. B, Opt. Phys., vol. 8, no. 5, pp. 1122-1126, May 1991.

[5] L. F. Mollenauer, S. G. Evangelides, and H. A. Haus, "Long-distance soliton propagation using lumped amplifiers and dispersion shifted fiber," J. Lightw. Technol., vol. 9, pp. 194-196, Feb. 1991.

[6] I. R. Gabitov and S. K. Turitsyn, "Averaged pulse dynamics in a cascaded transmission system with passive dispersion compensation," Opt. Lett., vol. 21, pp. 327-329, Mar. 1996.

[7] J.-C. Antona and S. Bigo, "Nonlinear cumulated phase as a criterion to assess performance of terrestrial WDM systems," in Proc. OFC '02, Anaheim, CA, Mar. 2002, pp. 365-367.

[8] A. Cauvin, Y. Frignac, and S. Bigo, "Single-channel nonlinear impairments at various bit-rate in dispersion-managed systems," in Proc. ECOC '03, Rimini, Italy, Sep. 2003, paper Mo4.2.4.

[9] A. Cauvin, Y. Frignac, and S. Bigo, "Nonlinear impairments at various bit rates in single-channel dispersion-managed systems," Electron. Lett., vol. 39, no. 23, pp. 1670-1671, Nov. 2003.

[10] B. Konrad and K. Petermann, "Optimum fiber dispersion in high-speed TDM systems," IEEE Photon. Technol. Lett., vol. 13, pp. 299-301, Apr. 2001 . 
[11] B. Konrad, K. Petermann, J. Berger, R. Ludwig, C. M. Weinert, H. G. Weber, and B. Schmauss, "Impact of fiber chromatic dispersion in high-speed TDM transmission systems," J. Lightw. Technol., vol. 20, pp. 2129-2135, Dec. 2002.

[12] H. Louchet, A. Hodzic, K. Petermann, A. Robinson, and R. Epworth, "Simple criterion for the characterization of nonlinear impairments in dispersion-managed optical transmission systems," IEEE Photon. Technol. Lett., vol. 17, pp. 2089-2091, Oct. 2005.

[13] R. I. Killey, H. J. Thiele, V. Mikhailov, and P. Bayvel, "Reduction of intrachannel nonlinear distortion in $40 \mathrm{~Gb} / \mathrm{s}$-based transmission over standard fiber," IEEE Photon. Technol. Lett., vol. 12, pp. 1624-1626, Dec. 2000

[14] A. Mecozzi, C. B. Clausen, and M. Shtaif, "System impact of intrachannel nonlinear effects in highly dispersed optical pulse transmission," IEEE Photon. Technol. Lett., vol. 12, pp. 1633-1635, Dec. 2000.

[15] Y. Frignac, J.-C. Antona, S. Bigo, and J.-P. Hamaide, "Numerical optimization of pre- and in-line dispersion compensation in dispersion managed systems at $40 \mathrm{~Gb} / \mathrm{s}$," in Proc. OFC '02, Anaheim, CA, Mar. 2002, pp. 612-613, paper ThFF5.

[16] Y. Frignac, J.-C. Antona, and S. Bigo, "Enhanced analytical engineering rule for fast optimization of dispersion map in $40 \mathrm{Gbit} / \mathrm{s}$-based transmission systems," in Proc. OFC '04, Los Angeles, CA, Feb. 2004, vol. 1, paper TuN3.

[17] Y. Frignac and S. Bigo, "Numerical optimization of residual dispersion in dispersion-managed systems at $40 \mathrm{Gbit} / \mathrm{s}$," in Proc. OFC '00, Los Angeles, CA, Feb. 2000, pp. 48-50, paper TuD3-1.

[18] P. Serena, A. Orlandini, and A. Bononi, "The memory of optimized dispersion-managed periodic optical links," in Proc. ECOC '07, Berlin, Germany, Sep. 2007, paper P.093.

[19] L. K. Wickham, R.-J. Essiambre, A. H. Gnauck, P. J. Winzer, and A. R. Chraplyvy, "Bit pattern dependence of intrachannel nonlinearities in pseudolinear transmission," IEEE Photon. Technol. Lett., vol. 16, pp. 1591-1593, Jun. 2004.

[20] P. Serena, A. Orlandini, and A. Bononi, "Fundamental laws of parametric gain in periodic dispersion-managed optical links," J. Opt. Soc. Amer. B, Opt. Phys., vol. 24, no. 4, pp. 773-787, Apr. 2007.

[21] M. J. Ablowitz, T. Hirooka, and T. Inoue, "Higher-order analysis of dispersion-managed transmission systems: Solutions and their characteristics," J. Opt. Soc. Amer. B, Opt. Phys., vol. 19, no. 12, pp. 2876-2885, Dec. 2002.

[22] G. P. Agrawal, Nonlinear Fiber Optics, 2nd ed. New York: Academic, 1995.

[23] A. V. T. Cartaxo, "Small-signal analysis for nonlinear and dispersive optical fibres, and its application to design of dispersion supported transmission systems with optical dispersion compensation," Proc. Inst. Elect. Eng. Optoelectron., vol. 146, pp. 213-222, Oct. 1999.

[24] A. Bononi, G. Bellotti, M. Varani, and C. Francia, "SPM/XPM-induced intensity distortion in WDM systems," in Optical Networking, A. Bononi, Ed. $\quad$ London, U.K.: Springer, 1999, pp. 383-398.

[25] X. Xiao, S. Gao, Y. Tian, and C. Yang, "Analytical optimization of the net residual dispersion in SPM-limited dispersion-managed systems," J. Lightw. Technol., vol. 24, pp. 2038-2043, May 2006.

[26] P. Serena, A. Orlandini, and A. Bononi, "Scaling laws for weakly-nonlinear WDM dispersion managed OOK systems," in Proc. ECOC '06, Cannes, France, Sep. 2006, paper We3.P.129.

[27] D. Zwillinger, Handbook of Differential Equations, 3rd ed. New York: Academic, 1997.

[28] O. V. Sinkin, R. Holzlöhner, J. Zweck, and C. R. Menyuk, "Optimization of the split-step fourier method in modeling optical-fiber communications systems," J. Lightw. Technol., vol. 21, pp. 61-68, Jan. 2003.

[29] E. Forestieri, "Evaluating the error probability in lightwave systems with chromatic dispersion, arbitrary pulse shape and post-detection filtering," J. Lightw. Technol., vol. 18, pp. 1493-1503, Nov. 2000.

[30] A. Vannucci, P. Serena, and A. Bononi, "The RP method: A new tool for the iterative solution of the nonlinear Schrödinger equation," $J$. Lightw. Technol., vol. 15, pp. 1102-1112, Jul. 2002.

[31] P. Serena, A. Orlandini, and A. Bononi, "A parametric-gain approach to the analysis of single-channel DPSK/DQPSK systems with nonlinear phase noise," J. Lightw. Technol., vol. 24, pp. 2026-2037, May 2006.

[32] X. Wey, "Power-weighted dispersion distribution function for characterizing nonlinear properties of long-haul optical transmission links," Opt. Lett., vol. 31, no. 17, pp. 2544-2546, Sep. 2006.

[33] M. J. Potasek, G. P. Agrawal, and S. C. Pinault, "Analytic and numerical study of pulse broadening in nonlinear dispersive optical fibers," $J$. Opt. Soc. Amer. B, Opt. Phys., vol. 3, no. 2, pp. 205-211, Feb. 1986.
[34] C. M. Bender and S. A. Orszag, Advanced Mathematical Methods for Scientists and Engineers. Asymptotic Methods and Perturbation Theory. New York: Springer-Verlag, 1999.

[35] P. Johannisson, "Interaction range and generation rate of nonlinear intrachannel signal distortion," J. Opt. Soc. Amer. B, Opt. Phys., vol. 24 no. 4, pp. 729-738, Apr. 2007.

[36] H. Louchet, K. Petermann, A. Robinson, and R. Epworth, "Introduction to the concept of nonlinear diffusion bandwidth," in Proc. ECOC '05, Glasgow, U.K., Sep. 2005, paper We4.P.011.

[37] R. A. Horn and C. R. Johnson, Matrix Analysis. Cambridge, U.K.: Cambridge Univ. Press, 1999.

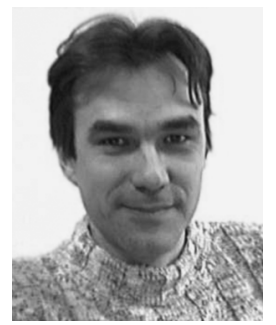

Alberto Bononi received the Laurea degree in electronics engineering (cum laude) from the University of Pisa, Italy, in 1988 and the M.A. and Ph.D. degrees in electrical engineering from Princeton University, Princeton, NJ, in 1992 and 1994, respectively.

Currently, he is an Associate Professor of Telecommunications with the School of Engineering, Università di Parma, Italy. He teaches courses in probability theory and stochastic processes, telecommunications networks, and optical communications. From 1994 to 1996, he was an Assistant Professor with the Electrical and Computer Engineering Department, State University of New York (SUNY), Buffalo, teaching courses in electric circuits and optical networks. In the summers of 1997 and 1999, he was a Visiting Faculty Member with the Département de Genie Électrique, Université Laval, QC, Canada, doing research on fiber amplifiers. His present research interests include system design and performance analysis of high-speed all-optical networks, nonlinear fiber transmission for WDM systems, linear and nonlinear polarization mode dispersion, and transient gain dynamics in optical amplifiers.

Prof. Bononi is an Associate Editor of the IEEE TRANSACTIONS ON COMMUNICATIONS.

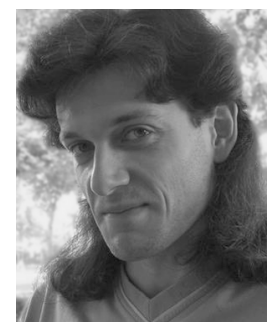

Paolo Serena was born in Piacenza, Italy, in 1973 $\mathrm{He}$ received the degree in electronic engineering and the $\mathrm{Ph} . \mathrm{D}$. degree in information technology from the Università degli Studi di Parma, Parma, Italy, in 1999 and 2003, respectively.

His doctoral work focused on the nonlinear interaction between noise and signal in optical systems. Currently, he is an Assistant Professor of telecommunications with the Department of Information Engineering, University of Parma, where he teaches a course on advanced optical communication and on fundamentals of communication systems. He is currently collaborating in research projects with Alcatel-Lucent (Nozay, France) on long-haul WDM systems. His main research interests include the parametric gain of the ASE noise by nonlinear effects, phase-shifted modulation formats, and performance optimization in the nonlinear regime.

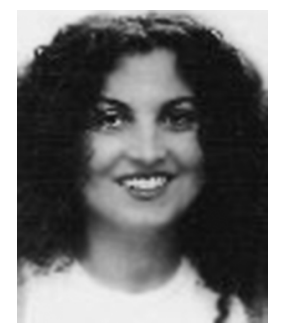

Alessandra Orlandini was born in Parma, Italy, in December 1973. She received the Laurea degree in electronics engineering (major in telecommunications) and the the Ph.D. degree in information technology from the University of Parma in 1998 and 2002, respectively.

In 2002, she joined the Optical Communication group, Department of Information Engineering, University of Parma, as a contract Researcher. She collaborated on research projects with Alcatel-Lucent (Nozay, France) on long-haul WDM systems. Her research interests were about optical differential phase modulation formats and the effect of phase noise in dispersion-managed WDM systems and also about the application of multicanonical Monte Carlo technique to BER measurement. She also studied polarization mode dispersion and its interaction with Kerr nonlinearities in WDM systems. She is the author or coauthor of more than 20 papers in international journals and conference proceedings. She is now with Custom Engineering, Parma, with her main interest being in the development of thermal printers and optical barcode readers. 